\title{
AN EFFICIENT REDUCED BASIS SOLVER FOR STOCHASTIC GALERKIN MATRIX EQUATIONS*
}

\author{
C. E. POWELL ${ }^{\dagger}$, D. SILVESTER ${ }^{\dagger}$, AND V. SIMONCINI ${ }^{\ddagger}$
}

\begin{abstract}
Stochastic Galerkin finite element approximation of PDEs with random inputs leads to linear systems of equations with coefficient matrices that have a characteristic Kronecker product structure. By reformulating the systems as multiterm linear matrix equations, we develop an efficient solution algorithm which generalizes ideas from rational Krylov subspace approximation. Our working assumptions are that the number of random variables characterizing the random inputs is modest, in the order of a few tens, and that the dependence on these variables is linear, so that it is sufficient to seek only a reduction in the complexity associated with the spatial component of the approximation space. The new approach determines a low-rank approximation to the solution matrix by performing a projection onto a low-dimensional space and provides an efficient solution strategy whose convergence rate is independent of the spatial approximation. Moreover, it requires far less memory than the standard preconditioned conjugate gradient method applied to the Kronecker formulation of the linear systems.
\end{abstract}

Key words. generalized matrix equations, PDEs with random data, stochastic finite elements, iterative solvers, rational Krylov subspace methods

AMS subject classifications. 35R60, 60H35, 65N30, 65F 10

DOI. $10.1137 / 15 \mathrm{M} 1032399$

1. Introduction. This paper is concerned with the design and implementation of efficient iterative solution algorithms for high-dimensional linear algebra systems that arise from Galerkin approximation of elliptic PDE problems with correlated random inputs. The tensor product structure of the Galerkin approximation space and the low-rank structure that is inherent in the discrete systems is exploited in our innovative solution strategy. This strategy builds on recent progress in rational Krylov subspace approximation (see, for example, Simoncini [24]) and generates an accurate reduced basis approximation of the spatial component of the Galerkin solution.

We focus on stochastic steady-state diffusion equations with homogeneous Dirichlet boundary conditions. To this end, let $D \subset \mathbb{R}^{2}$ be a sufficiently regular spatial domain and let $\Omega$ be a sample space associated with a probability space $(\Omega, \mathcal{F}, \mathbb{P})$. Our goal is to approximate $u: D \times \Omega \rightarrow \mathbb{R}$ such that $\mathbb{P}$-a.s.,

$$
\begin{aligned}
-\nabla \cdot(a(\vec{x}, \omega) \nabla u(\vec{x}, \omega)) & =f(\vec{x}) & & \text { in } D, \\
u(\vec{x}, \omega) & =0 & & \text { on } \partial D .
\end{aligned}
$$

We assume that $f$ is deterministic and $a$ is a random field that can be expressed as a linear function of a finite number of real-valued independent random variables $\xi_{r}: \Omega \rightarrow \Gamma_{r} \subset \mathbb{R}$ of the form

$$
a(\vec{x}, \omega)=a_{0}(\vec{x})+\sum_{r=1}^{m} a_{r}(\vec{x}) \xi_{r}(\omega) .
$$

*Submitted to the journal's Methods and Algorithms for Scientific Computing section July 24, 2015; accepted for publication (in revised form) October 25, 2016; published electronically January 10, 2017.

http://www.siam.org/journals/sisc/39-1/M103239.html

†School of Mathematics, University of Manchester, Oxford Road, Manchester M13 9PL, United Kingdom (c.powell@manchester.ac.uk,d.silvester@manchester.ac.uk).

${ }^{\ddagger}$ Dipartimento di Matematica, Università di Bologna, Piazza di Porta S. Donato 5, I-40127 Bologna, Italy, and IMATI-CNR, Pavia, Italy (valeria.simoncini@unibo.it).

A141 
A common choice is a truncated Karhunen-Loève (KL) expansion (see Lord, Powell, and Shardlow [15] for further discussion). In that case,

$$
a(\vec{x}, \omega)=\mu(\vec{x})+\sigma \sum_{r=1}^{m} \sqrt{\lambda_{r}} \phi_{r}(\vec{x}) \xi_{r}(\omega)
$$

where $\mu$ is the expected value of the diffusion coefficient, $\sigma$ is the standard deviation, and $\left\{\lambda_{r}, \phi_{r}\right\}$ are eigenpairs of the integral operator $\mathcal{B}$ associated with $B\left(\vec{x}_{1}, \vec{x}_{2}\right)=$ $\left(1 / \sigma^{2}\right) C\left(\vec{x}_{1}, \vec{x}_{2}\right)$, where $C: D \times D \rightarrow \mathbb{R}$ is the covariance function. We assume $\lambda_{1} \geq$ $\lambda_{2} \geq \cdots$ so that the terms retained in (1.3) correspond to the $m$ largest eigenvalues of the covariance.

Weak formulations of (1.1) have been well studied in the literature (e.g., see [15, Chapter 9]) and well-posedness follows straightforwardly from the Lax-Milgram lemma if realizations of $a$ are positive and bounded. To ensure this, we restrict our attention to independent uniform random variables $\xi_{r}$ on $\Gamma_{r}=[-1,1]$ and assume that there exist constants $a_{0}^{\min }$ and $a_{0}^{\max }$ satisfying

$$
0<a_{0}^{\min } \leq a_{0}(\vec{x}) \leq a_{0}^{\max }<\infty \quad \text { a.e. in } D
$$

and the coefficient functions $a_{r}$ satisfy

$$
\sum_{r=1}^{m}\left\|a_{r}\right\|_{\infty}<a_{0}^{\min }
$$

Stochastic Galerkin methods (introduced by Babuška and collaborators [1], [4]) seek approximations $u_{h p}$ to the weak solution $u$ of (1.1) in finite-dimensional approximation spaces of the form $Z_{h} \otimes S_{p}$, where $Z_{h} \subset H_{0}^{1}(D)$ and $S_{p} \subset L_{\varrho}^{2}(\Gamma)$. As usual, $H_{0}^{1}(D)$ is the Sobolev space associated with $D$, and $L_{\varrho}^{2}(\Gamma)$ denotes the set of functions $v$ on $\Gamma:=\Gamma_{1} \times \cdots \times \Gamma_{m}=[-1,1]^{m}$ satisfying

$$
\langle v, v\rangle_{\varrho}:=\int_{\Gamma} \varrho(\mathbf{y}) v(\mathbf{y})^{2} d \mathbf{y}<\infty,
$$

where $\varrho(\mathbf{y})=2^{-m}$ is the joint density of $\left[\xi_{1}, \ldots, \xi_{m}\right]$ and $\mathbf{y}=\left[y_{1}, \ldots, y_{m}\right]$ with $y_{r}:=\xi_{r}(\omega)$. We choose $Z_{h}=\operatorname{span}\left\{\varphi_{1}(\vec{x}), \ldots, \varphi_{n_{x}}(\vec{x})\right\}$ to be a finite element space associated with a spatial mesh of $D$ with characteristic element size $h$. In our experiments, we choose $S_{p}=\operatorname{span}\left\{\psi_{1}(\mathbf{y}), \ldots, \psi_{n_{\xi}}(\mathbf{y})\right\}$ to be the set of multivariate polynomials of total degree $p$ or less in $y_{1}, \ldots, y_{m}$ on $\Gamma$. However, tensor product polynomials could also be used. For the first choice, we have

$$
n_{\xi}=\operatorname{dim}\left(S_{p}\right)=\frac{(m+p) !}{m ! p !}
$$

and, as usual (see [15, Chapter 9]), we construct the basis functions as

$$
\psi_{j}(\mathbf{y})=\prod_{s=1}^{m} \psi_{j_{s}}\left(y_{s}\right), \quad j=\left(j_{1}, \ldots, j_{m}\right), \quad \sum_{s=1}^{m} j_{s} \leq p
$$

where $\left\{\psi_{j_{s}}, j_{s}=0,1,2, \ldots, p\right\}$ are univariate Legendre polynomials of degree $j_{s}$ that are orthonormal on $[-1,1]$ with respect to the weight function $\varrho_{s}=1 / 2$. This yields a basis of multivariate Legendre polynomials that are orthonormal with respect to $\varrho(\mathbf{y})=\varrho_{1}\left(y_{1}\right) \cdots \varrho_{m}\left(y_{m}\right)$. 
The structure of the expansion (1.2) and the tensor product form of the approximation space $Z_{h} \otimes S_{p}$ leads to a structured linear algebraic system $\mathcal{A} \mathbf{x}=\mathbf{b}$ of $n_{x} \cdot n_{\xi}$ equations where

$$
\mathcal{A}=G_{0} \otimes K_{0}+\sum_{r=1}^{m} G_{r} \otimes K_{r}, \quad \mathbf{b}=\mathbf{g}_{0} \otimes \mathbf{f}_{0}
$$

and the vector $\mathbf{x}$ contains the coefficients in the expansion of the stochastic Galerkin approximation in the tensor product basis $\left\{\varphi_{i} \psi_{j}, i=1, \ldots, n_{x}, j=1, \ldots, n_{\xi}\right\}$. In (1.7), the symbol $\otimes$ denotes the Kronecker product. $K_{0}$ and $K_{r}$ are finite element stiffness matrices of size $n_{x} \times n_{x}$ defined by

$$
\left[K_{0}\right]_{i, j}=\int_{D} a_{0} \nabla \varphi_{i} \cdot \nabla \varphi_{j} d \vec{x}, \quad\left[K_{r}\right]_{i, j}=\int_{D} a_{r} \nabla \varphi_{i} \cdot \nabla \varphi_{j} d \vec{x}, \quad r=1, \ldots, m,
$$

for $i, j=1, \ldots, n_{x}$. The matrices $G_{0}$ and $G_{r}$ are of size $n_{\xi} \times n_{\xi}$ and are defined by

$$
\left[G_{0}\right]_{s, t}=\left\langle\psi_{s}, \psi_{t}\right\rangle_{\varrho}, \quad\left[G_{r}\right]_{s, t}=\left\langle y_{r} \psi_{s}, \psi_{t}\right\rangle_{\varrho}, \quad r=1, \ldots, m,
$$

where $\psi_{s}, \psi_{t}, s, t=1, \ldots, n_{\xi}$ are basis functions for $S_{p}$. Since we choose these to be orthonormal with respect to $\langle\cdot, \cdot\rangle_{\varrho}, G_{0}=I$. The vector $\mathbf{g}_{0}$ is the first column of $G_{0}$, and $\mathbf{f}_{0}$ is the vector associated with the finite element discretization of the deterministic analogue of (1.1). That is,

$$
\left[\mathbf{f}_{0}\right]_{i}=\int_{D} f \varphi_{i} d \vec{x}, \quad i=1, \ldots, n_{x}
$$

From now on, we will refer to the algebraic linear system $\mathcal{A} \mathbf{x}=\mathbf{b}$ as the Kronecker formulation of the discrete problem. Alternatively, by introducing the solution matrix

$$
X=\left[\mathbf{x}_{1}, \mathbf{x}_{2}, \ldots, \mathbf{x}_{n_{\xi}}\right] \in \mathbb{R}^{n_{x} \times n_{\xi}},
$$

whose $j$ th column is the subvector of $\mathbf{x}$ containing the coefficients associated with the $j$ th basis function $\psi_{j}$ for $S_{p}$, the linear system can also be rewritten as a linear multiterm matrix equation

$$
K_{0} X G_{0}^{\top}+\sum_{r=1}^{m} K_{r} X G_{r}^{\top}=F .
$$

For example, see [13, Lemma 4.3.1]. By introducing the operator vec $(\cdot)$, which stacks the columns of a matrix one after the other to form a vector, we have the relation $\mathbf{x}=\operatorname{vec}(X)$. The $n_{x} \times n_{\xi}$ matrix $F$ satisfies $\operatorname{vec}(F)=\mathbf{b}$ or, equivalently, $F=\mathbf{f}_{0} \mathbf{g}_{0}^{\top}$. We will refer to (1.8) as the matrix equation formulation of the discrete problem.

There is a substantial body of work on solving the Kronecker formulation of discrete problems associated with stochastic Galerkin approximation of elliptic PDEs. Since $\mathcal{A}$ is sparse, symmetric, and positive definite, the standard conjugate gradient (CG) method can be applied. However, this must be done in a smart way without assembling $\mathcal{A}$. (See, e.g., [10] for an early reference.) Matrix-vector products should be done by exploiting the relation

$$
\sum_{r=0}^{m}\left(G_{r} \otimes K_{r}\right) \mathbf{v}=\operatorname{vec}\left(\sum_{r=0}^{m} K_{r} V G_{r}^{\top}\right), \quad V=\operatorname{array}(\mathbf{v}),
$$


where array (.) forms a matrix $V$ of size $n_{x} \times n_{\xi}$ from the $n_{\xi}$ subvectors of the given vector v. Preconditioners that exploit the structure of $\mathcal{A}$ are another essential ingredient (see, for example, [19], [26], [25], [21], [8]). In particular, the mean-based preconditioner $P=G_{0} \otimes K_{0}$ analyzed by Powell and Elman in [19] requires $n_{\xi}$ inexact decoupled solves with $K_{0}$ in each CG iteration. Mean-based preconditioning is often used in practice due to its nonintrusive nature, despite its lack of robustness when we increase the ratio $\sigma / \mu$ in the KL expansion (1.3).

We will focus on the matrix equation formulation (1.8) in this paper. In particular, we propose a new method that determines a low-rank approximation to the solution matrix $X$ by performing a projection onto a subspace of dimension $n_{k} \cdot n_{\xi}$, where $n_{k}$ is much smaller than $n_{x}$. In analogy to what is done in reduced basis approaches to nonintrusive methods (for example, see [20]), our strategy only attempts to achieve a reduction in the complexity associated with the high dimension of the deterministic part of the problem. Hence our working assumption is that the number of terms $m$ in (1.2) is modest (in the low tens) so that the dimensions of the stochastic Galerkin spaces $S_{p}$ and $Z_{h}$ satisfy $n_{\xi} \ll n_{x}$. Our philosophy is that long vectors of size $n_{x} \cdot n_{\xi}$ should never be constructed in generating a low-rank approximation. Previous work on low-rank approximation methods has focussed on finding the dominant eigenvectors of (KL-like) expansions of the solution matrix (see, for example, Matthies and Keese [17]). There has also been significant recent research on the construction of low-rank tensor decompositions of the discrete solution; in particular, by Khoromskij and Schwab [14], Matthies and Zander [18], and Ballani and Grasedyck [2]. The novel aspect of our strategy is that a reduced approximation space is built up on-the-fly from rational Krylov subspaces. A projection is then used to obtain a reduced matrix equation whose solution yields the low-rank approximation. We note that tensor methods are to be recommended when tensor product polynomials are used and $m$, and hence the stochastic dimension $n_{\xi}$, are extremely large.

An outline of the paper is as follows. The construction of reduced rational Krylov approximation spaces for multiterm linear matrix equations is discussed in general terms in the next section. In section 3, we discuss the model problem (1.1) and modify the associated stochastic Galerkin matrix equations so that the general algorithm from section 2 can be applied efficiently. Details of our implementation are given in section 4. Finally, the performance of the resulting solver is assessed for two test problems in section 5. Our experiments demonstrate that huge computational savings - compared to solving the Kronecker formulation of the matrix equations using standard preconditioned CG - can be achieved when the dimension $n_{x} \cdot n_{\xi}$ is large. That is, when one is looking to compute an accurate approximation to the solution of (1.1) on a fine spatial mesh when the coefficient (1.2) is a function of up to $m=20$ random variables.

2. Reduced rational Krylov approximation. Krylov-type subspaces have proved to be effective approximation spaces in projection methods for linear matrix equations; see [24]. A shortcoming of classical (polynomial in the coefficient matrix A) Krylov subspaces is that they may require a large dimension to satisfactorily approximate the sought after solution. The use of rational Krylov subspaces, which involve rational matrix functions of the type $\left(A-s_{j} I\right)^{-1}, s_{j} \in \mathbb{C}$, have practically solved this problem in many applications. At the cost of solving a linear system at each iteration, the generated space quickly builds up spectral information of the matrix $A$, thus allowing a good approximation in a space of lower dimension than classical Krylov spaces. Rational Krylov subspaces with appropriately chosen parameters $s_{j}$ 
have thus become a standard in the solution of large scale matrix equations and other problems such as matrix function evaluations. We propose an extension of the rational Krylov subspace idea to solve (1.8). The presence of several coefficient matrices makes the generalization very challenging. Moreover, the need for a small though rich (i.e., spectrally informative) approximation space is particularly pressing.

To introduce the concept of reduced rational Krylov approximation, suppose that we are given a set of $m$ sparse symmetric positive definite matrices $A_{1}, \ldots, A_{m}$ of size $n_{x} \times n_{x}$, whose spectra are all contained in a small interval $\mathcal{S} \subset \mathbb{R}^{+}$. The significance of $\mathcal{S}$ will become apparent later. In addition, let $A_{0}=I$ be the $n_{x} \times n_{x}$ identity matrix. Now suppose that we wish to solve a general multiterm matrix equation of the form

$$
A_{0} X B_{0}+A_{1} X B_{1}+\cdots+A_{m} X B_{m}=\mathbf{f}_{0} \mathbf{g}_{0}^{\top}
$$

for the unknown matrix $X$ of size $n_{x} \times n_{\xi}$ (with $n_{\xi} \ll n_{x}$ ), where the matrices $B_{r}$, $r=0,1, \ldots, m$ are of size $n_{\xi} \times n_{\xi}$, and the vectors $\mathbf{f}_{0}$ and $\mathbf{g}_{0}$ are of length $n_{x}$ and $n_{\xi}$ respectively. In this section, we simply view (2.1) as an abstract problem and temporarily leave aside the specific details of stochastic Galerkin matrix equations.

Suppose that we can generate an orthonormal basis for an approximation space $\mathcal{K}_{k} \subset \mathbb{R}^{n_{x}}$ of dimension $n_{k} \ll n_{x}$. If we collect the $n_{k}$ basis vectors into a matrix $V_{k}$ of size $n_{x} \times n_{k}$, then an approximate solution to (2.1) can be sought in the form $X_{k}=V_{k} Y_{k} \approx X$, where the $n_{k} \times n_{\xi}$ matrix $Y_{k}$ (the reduced solution matrix) can be defined by insisting that the residual

$$
R_{k}:=X_{k} B_{0}+A_{1} X_{k} B_{1}+\cdots+A_{m} X_{k} B_{m}-\mathbf{f}_{0} \mathbf{g}_{0}^{\top}
$$

satisfies the Galerkin condition $V_{k}^{\top} R_{k}=0$. This may be viewed as an orthogonality condition for each column of the matrix $R_{k}$ or as a matrix orthogonality condition in the sense that

$$
\operatorname{vec}\left(V_{k}^{\top} R_{k}\right)=\left(I \otimes V_{k}^{\top}\right) \mathbf{r}=\mathbf{0},
$$

where $\mathbf{r}=\operatorname{vec}\left(R_{k}\right)$. Substituting $X_{k}=V_{k} Y_{k}$ into this expression we see that $Y_{k}$ is the solution of the reduced (or projected) matrix equation

$$
(\underbrace{V_{k}^{\top} V_{k}}_{I}) Y_{k} B_{0}+(\underbrace{V_{k}^{\top} A_{1} V_{k}}_{\bar{A}_{1}}) Y_{k} B_{1}+\cdots+(\underbrace{V_{k}^{\top} A_{m} V_{k}}_{\bar{A}_{m}}) Y_{k} B_{m}=\underbrace{V_{k}^{\top} \mathbf{f}_{0}}_{\overline{\mathbf{f}_{0}}} \mathbf{g}_{0}^{\top} .
$$

Comparing (2.2) with (2.1) we see that the left matrices have been reduced in size (from $n_{x} \times n_{x}$ to $n_{k} \times n_{k}$ ) but will be dense rather than sparse. The right matrices $B_{r}$ are unchanged. Readers who are more familiar with the Kronecker formulation of matrix equations will note that (2.2) is equivalent to the algebraic system $\mathcal{A}_{k} \mathbf{y}_{k}=\mathbf{b}_{k}$, where $\mathbf{y}_{k}=\operatorname{vec}\left(Y_{k}\right)$ and

$$
\mathcal{A}_{k}=B_{0} \otimes I+\sum_{r=1}^{m} B_{r} \otimes \bar{A}_{r}, \quad \mathbf{b}_{k}=\mathbf{g}_{0} \otimes \overline{\mathbf{f}_{0}} .
$$

To effectively implement such a strategy we will need to answer two questions: (i) how do we construct the approximation space $\mathcal{K}_{k}$, keeping $n_{k}$ as small as possible while still maintaining accuracy? and (ii) can we solve the reduced matrix equation (2.2) for $Y_{k}$ efficiently? For the latter, we will consider the Kronecker formulation (2.3). We can compute $\mathbf{y}_{k}$ using a direct method if $n_{k} \cdot n_{\xi}$ is small or else use a matrix-oriented 
iterative solver such as CG, taking care to ensure that matrix-vector products are performed efficiently using (1.9). For the former, we want to develop an iterative procedure for building a sequence of approximation spaces $\mathcal{K}_{1} \subset \mathcal{K}_{2} \subset \cdots \subset \mathcal{K}_{j} \subset \cdots$ that terminates after $k$ iterations when $\mathcal{K}_{k}$ is judged to be rich enough. To achieve this objective, starting from the initial (scaled) vector ${ }^{1} \mathbf{v}_{0}=\mathbf{f}_{0} /\left\|\mathbf{f}_{0}\right\| \in \mathbb{R}^{n_{x}}$ and given a vector of real positive parameters $\mathbf{S}=\left[s_{1}, s_{2}, \ldots\right]$, we will iteratively generate spaces $\mathcal{K}_{j}, j=1,2, \ldots$ of increasing dimension $n_{j}$ that are built from a nested sequence of rational Krylov approximation spaces. We give a brief outline of the construction of these spaces next. All other implementation details are deferred to section 4 .

At the first iteration $j=1$, we start with $V_{0}=\mathbf{v}_{0}$ and compute

$$
W=\left[\left(A_{1}+s_{1} I\right)^{-1} \mathbf{v}_{0}, \ldots,\left(A_{m}+s_{1} I\right)^{-1} \mathbf{v}_{0}\right] \in \mathbb{R}^{n_{x} \times m} .
$$

Next, we identify the $\ell_{1}$ most significant directions of this matrix. This is done by computing the singular values $\sigma_{i}$ of $W$ and retaining only the $\ell_{1}$ left singular vectors $\mathbf{u}_{i}, i=1, \ldots, \ell_{1}$ associated with the values that contribute a fixed proportion $\beta \%$ of the total (typically $\beta=99$ ). That is, we choose $\ell_{1}$ such that $\sum_{i=1}^{\ell_{1}} \sigma_{i}>\frac{\beta}{100} \sum_{i=1}^{m} \sigma_{i}$. The approximation space $\mathcal{K}_{1}$ will be of dimension $n_{1}=\ell_{1}+1 \leq m+1$ and is given by range $\left(V_{1}\right)$ once the vectors have been modified to form an orthonormal basis

$$
V_{1}=\operatorname{orth}\left(\left[\mathbf{v}_{0}, \mathbf{u}_{1}, \ldots, \mathbf{u}_{\ell_{1}}\right]\right)=\left[\mathbf{v}_{0}, \mathbf{v}_{1}, \ldots, \mathbf{v}_{\ell_{1}}\right] \in \mathbb{R}^{n_{x} \times\left(\ell_{1}+1\right)} .
$$

The second iteration is defined by computing a new matrix

$$
W=\left[\left(A_{1}+s_{2} I\right)^{-1} \mathbf{v}_{1}, \ldots,\left(A_{m}+s_{2} I\right)^{-1} \mathbf{v}_{1}\right]
$$

and then computing its singular value decomposition in order to determine which $\ell_{2}$ directions should be kept. The approximation space $\mathcal{K}_{2}$ has dimension $n_{2}=\ell_{2}+\ell_{1}+$ $1 \leq 2 m+1$. At the $j$ th iteration, the $j$ th column $\mathbf{v}_{j-1}$ of the basis matrix $V_{j-1}$ is used to expand the space. The procedure continues to enrich the space until, after $k$ steps, an appropriate convergence criterion is satisfied. The orthogonality property $V_{k}^{\top} R_{k}=0$ ensures finite termination in exact arithmetic, since the exact solution will be determined in an approximation space of dimension at most $n_{x}$. However, a good approximation is clearly sought in a space with a much smaller dimension.

The vector of positive parameters $\mathbf{s}=\left[s_{1}, s_{2}, \ldots\right]$ is selected before the iteration begins. Notice that since each $A_{r}$ is symmetric and positive definite, each of the shifted matrices $\left(A_{r}+s_{j} I\right), r=1, \ldots, m$ is invertible, regardless of the particular positive value of $s_{j}$ chosen. We delay a discussion of specific choices for $s_{j}$ until section 4.2. Here, we simply note that we apply the same parameter $s_{j}$ when computing each of the $m$ columns of $W$. In this setting, the user must supply only one parameter $s_{j}$ at the $j$ th iteration. Of course, the number $k$ of required iterations will not be known in advance so we may cycle through a fixed number of chosen values, say, $\mathbf{s}=\left[s_{1}, s_{2}, \ldots, s_{10}\right]$. For more general multiterm matrix equations, up to $m$ different parameters could be employed in each iteration. However, we will not consider that case here. Depending on the problem, further simplifications may be possible. For instance, it might be appropriate to choose a single value of $s_{j}$ in all iterations. We will discuss multiple-parameter and parameter-free strategies in section 4.2 and investigate them numerically in section 5 . For now, we use the subscript $j$ to allow for the possibility of dynamically updating $s_{j}$ as the iteration proceeds.

\footnotetext{
${ }^{1}$ The vector $\mathbf{f}_{0}$ is the initial residual associated with the deterministic algebraic system and a zero initial guess.
} 
To describe the Krylov approximation space generated, let

$$
\mathbb{K}_{\ell}\left(A_{r}, \mathbf{v}_{0}, \mathbf{s}\right):=\operatorname{span}\left\{\mathbf{v}_{0},\left(A_{r}+s_{r_{1}} I\right)^{-1} \mathbf{v}_{0}, \ldots, \prod_{j=1}^{\ell}\left(A_{r}+s_{r_{j}} I\right)^{-1} \mathbf{v}_{0}\right\}
$$

be the rational Krylov subspace associated with the matrix $A_{r}$, the initial vector $\mathbf{v}_{0}$, and the chosen vector of parameters $\mathbf{s}$, where $\left[s_{r_{1}}, s_{r_{2}}, \ldots, s_{r_{\ell}}\right]$ contains an appropriate subset of length $\ell$ of the components of $\mathbf{s}$. (The parameters appear in different orders and combinations for each $r=1, \ldots, m$.) After $k$ iterations, the approximation space contains the rational Krylov subspaces $\mathbb{K}_{\ell}\left(A_{r}, \mathbf{v}_{0}, \mathbf{s}\right), r=1, \ldots, m$ for some particular values of $\ell$ (which may be different for each $r$ ). However, it is actually a richer space, in the sense that it also contains combinations of rational functions in the coefficient matrices $\left\{A_{1}, \ldots, A_{m}\right\}$ that are not included in those subspaces. This way, we expect to construct a smaller subspace than by simply taking the union of individual rational spaces. As an illustration, consider the specific case of $m=3$. Suppose we perform three iterations and choose distinct parameters $s_{1}, s_{2}$, and $s_{3}$. The process generates the following space (where, for ease of exposition, we do not orthogonalize or deflate the vectors):

$$
\begin{aligned}
& \operatorname{span}\left\{\mathbf{v}_{0},\right. \underbrace{\left(A_{1}+s_{1} I\right)^{-1} \mathbf{v}_{0}}_{=: \mathbf{v}_{1}}, \underbrace{\left(A_{2}+s_{1} I\right)^{-1}}_{=: \mathbf{v}_{2}} \mathbf{v}_{0}, \underbrace{\left(A_{3}+s_{1} I\right)^{-1}}_{=: \mathbf{v}_{3}} \mathbf{v}_{0}, \\
&\left(A_{1}+s_{2} I\right)^{-1} \mathbf{v}_{1},\left(A_{2}+s_{2} I\right)^{-1} \mathbf{v}_{1},\left(A_{3}+s_{2} I\right)^{-1} \mathbf{v}_{1}, \\
&\left.\left(A_{1}+s_{3} I\right)^{-1} \mathbf{v}_{2},\left(A_{2}+s_{3} I\right)^{-1} \mathbf{v}_{2},\left(A_{3}+s_{3} I\right)^{-1} \mathbf{v}_{2}\right\} \\
&=\operatorname{span}\left\{\mathbf{v}_{0},\left(A_{1}+s_{1} I\right)^{-1} \mathbf{v}_{0},\left(A_{2}+s_{1} I\right)^{-1} \mathbf{v}_{0},\left(A_{3}+s_{1} I\right)^{-1} \mathbf{v}_{0},\right. \\
&\left(A_{1}+s_{2} I\right)^{-1}\left(A_{1}+s_{1} I\right)^{-1} \mathbf{v}_{0},\left(A_{2}+s_{2} I\right)^{-1}\left(A_{1}+s_{1} I\right)^{-1} \mathbf{v}_{0},\left(A_{3}+s_{2} I\right)^{-1} \\
&\left(A_{1}+s_{1} I\right)^{-1} \mathbf{v}_{0},\left(A_{1}+s_{3} I\right)^{-1}\left(A_{2}+s_{1} I\right)^{-1} \mathbf{v}_{0},\left(A_{2}+s_{3} I\right)^{-1}\left(A_{2}+s_{1} I\right)^{-1} \\
&\left.\mathbf{v}_{0},\left(A_{3}+s_{3} I\right)^{-1}\left(A_{2}+s_{1} I\right)^{-1} \mathbf{v}_{0},\right\},
\end{aligned}
$$

which consists of rational functions in $\left\{A_{1}, A_{2}, A_{3}\right\}$. We see that the space contains the subspaces

$$
\begin{aligned}
& \mathbb{K}_{2}\left(A_{1}, \mathbf{v}_{0}, \mathbf{s}\right)=\operatorname{span}\left\{\mathbf{v}_{0},\left(A_{1}+s_{1} I\right)^{-1} \mathbf{v}_{0},\left(A_{1}+s_{2} I\right)^{-1}\left(A_{1}+s_{1} I\right)^{-1} \mathbf{v}_{0}\right\}, \\
& \mathbb{K}_{2}\left(A_{2}, \mathbf{v}_{0}, \mathbf{s}\right)=\operatorname{span}\left\{\mathbf{v}_{0},\left(A_{2}+s_{1} I\right)^{-1} \mathbf{v}_{0},\left(A_{2}+s_{3} I\right)^{-1}\left(A_{2}+s_{1} I\right)^{-1} \mathbf{v}_{0}\right\},
\end{aligned}
$$

associated with $A_{1}$ and $A_{2}$ and the subspace

$$
\mathbb{K}_{1}\left(A_{3}, \mathbf{v}_{0}, \mathbf{s}\right)=\operatorname{span}\left\{\mathbf{v}_{0},\left(A_{3}+s_{1} I\right)^{-1} \mathbf{v}_{0}\right\},
$$

associated with $A_{3}$. However, the four vectors

$$
\begin{array}{lll}
\left(A_{2}+s_{2} I\right)^{-1}\left(A_{1}+s_{1} I\right)^{-1} \mathbf{v}_{0}, & \left(A_{3}+s_{2} I\right)^{-1}\left(A_{1}+s_{1} I\right)^{-1} \mathbf{v}_{0}, \\
\left(A_{1}+s_{3} I\right)^{-1}\left(A_{2}+s_{1} I\right)^{-1} \mathbf{v}_{0}, & \left(A_{3}+s_{3} I\right)^{-1}\left(A_{2}+s_{1} I\right)^{-1} \mathbf{v}_{0}
\end{array}
$$

are not contained in any of these individual subspaces.

Remark 2.1. The low-rank projection method described above may be viewed as a generalization of the standard Galerkin method applied to the Sylvester equation

$$
A X+X G=\mathbf{f g}^{\top},
$$

Copyright $@$ by SIAM. Unauthorized reproduction of this article is prohibited. 
where the dimension of $G$ is much smaller than that of $A$. The analogy with our strategy is that a projection is only performed on the "large" part of the equation. The smaller right-looking part (involving $G$ ) is not reduced. See Simoncini [24] for a complete survey.

3. Application to stochastic Galerkin matrix equations. Let us now return to the multiterm matrix equation (1.8) associated with stochastic Galerkin approximations of (1.1),

$$
K_{0} X G_{0}^{\top}+\sum_{r=1}^{m} K_{r} X G_{r}^{\top}=\mathbf{f}_{0} \mathbf{g}_{0}^{\top} .
$$

Recall from section 1 that each term corresponds to a different term in the expansion of the diffusion coefficient (1.2). The left matrices $K_{r}, r=0,1, \ldots, m$ are symmetric stiffness matrices of size $n_{x} \times n_{x}$. The right matrices $G_{r}, r=0,1, \ldots, m$ are of size $n_{\xi} \times n_{\xi}$ and are also symmetric. Due to the constuction of the basis for $S_{p}$ we have $G_{0}=I$. In addition, $G_{1}, \ldots, G_{m}$ all have at most two nonzero entries per row. Since we are working with uniform random variables on $\Gamma_{r}=[-1,1]$, it can also be shown that the eigenvalues of $G_{r}$ are contained in $[-1,1]$ for $r=1,2, \ldots, m$. That is, $G_{1}, \ldots, G_{m}$ are all indefinite and have spectral bounds that do not depend on the polynomial degree $p$ or the number of random variables $m$. See Powell and Elman [19] and Ernst and Ullmann [9] for further discussion. Next, since $\mathbf{g}_{0}$ is the first column of $G_{0}$, we have

$$
F=\mathbf{f}_{0} \mathbf{g}_{0}^{T}=\left[\mathbf{f}_{0}, \mathbf{0}, \ldots, \mathbf{0}\right] \in \mathbb{R}^{n_{x} \times n_{\xi}},
$$

so that only the first column of the matrix $F$ on the right-hand side of (3.1) is nonzero.

The matrix equation (3.1) has the same structure as (2.1), but the left matrices are generally indefinite. This is an issue for two reasons. On the one hand, positive definiteness of the left matrices ensures that the rational Krylov subspaces generated in section 2 are well-defined (the coefficient matrices used in the construction of $W$ are nonsingular); on the other hand, it ensures that the projected (reduced) matrices $V_{k}^{\top} A_{r} V_{k}$ in (2.2) are nonsingular. The matrix $K_{0}$ is always positive definite due to (1.4). However, $K_{1}, \ldots, K_{m}$ are indefinite whenever the functions $a_{1}, \ldots, a_{m}$ are not strictly positive. We encounter this situation when working with KL expansions (1.3) where $a_{r}:=\sigma \sqrt{\lambda_{r}} \phi_{r}$ and $\phi_{r}$ is an eigenfunction of a covariance operator. Spectral bounds for $K_{r}, r=0,1, \ldots, m$ are given in [19], and these depend on the finite element mesh parameter $h$ in an unfavorable way. To generate a matrix equation (2.1) that has positive definite left matrices whose spectral intervals do not grow as $h \rightarrow 0$, we will need to make two modifications to (3.1).

First, we formally divide the matrix system in (3.1) by $K_{0}$. This corresponds to applying the preconditioner $P=I \otimes K_{0}$ to (1.7). Since $K_{0}$ is symmetric and positive definite, we may exploit the Cholesky factorization $K_{0}=L L^{\top}$. Multiplying on the left in (3.1) by $L^{-1}$, defining $\widehat{X}:=L^{T} X$, and using $G_{0}=I$ and $G_{r}^{\top}=G_{r}$ yields the modified matrix equation

$$
\widehat{X}+\sum_{r=1}^{m} \widehat{K}_{r} \widehat{X} G_{r}=\widehat{\mathbf{f}}_{0} \mathbf{g}_{0}^{\top}
$$

where $\widehat{\mathbf{f}}_{0}:=L^{-1} \mathbf{f}_{0}$ and $\widehat{K}_{r}:=L^{-1} K_{r} L^{-T}$ for $r=1, \ldots, m$. When $K_{r}$ is indefinite $\widehat{K}_{r}$ is also indefinite. The eigenvalues of $\widehat{K}_{r}$ coincide with those of $K_{0}^{-1} K_{r}$, and bounds for these are established in [19, Lemma 3.4] in the case where the diffusion coefficient 
is given by (1.3). Generalizing this result to (1.2) and assuming for simplicity now that $a_{0}>0$ is a constant, it is straightforward to show that the eigenvalues of $\widehat{K}_{r}$ lie in the bounded interval $\left[-\tau_{r}, \tau_{r}\right]$, where

$$
\tau_{r}:=a_{0}^{-1}\left\|a_{r}\right\|_{L^{\infty}(D)} .
$$

If $a_{r}(\vec{x})>\nu_{r}>0$ for all $\vec{x} \in D$, so that $K_{r}$ is positive definite, then a tighter bound for the eigenvalues of $\widehat{K}_{r}$ is given by $\left[a_{0}^{-1} \nu_{r}, \tau_{r}\right]$, which is a subset of $\left[-\tau_{r}, \tau_{r}\right]$. The key point is that these spectral bounds do not depend on the finite element mesh parameter $h$.

Next, we want to find positive shifts $\alpha_{r}$ so that $\widehat{K}_{r}+\alpha_{r} I$ for $r=1, \ldots, m$ are positive definite. Using [19, Lemma 3.4], we know that the eigenvalues of $\widehat{K}_{r}+\alpha_{r} I$ lie in the interval

$$
\mathcal{S}_{r}:=\left[\alpha_{r}-\tau_{r}, \alpha_{r}+\tau_{r}\right],
$$

(although this bound is not tight if $a_{r}$ is strictly positive on $D$ ). If (1.5) holds, then we have $\sum_{r=1}^{m} \tau_{r}<1$ and thus $\tau_{r}<1$ for each $r=1,2, \ldots, m$. Hence, in our problems, choosing $\alpha_{r}=1$ for $r=1, \ldots, m$ suffices.

To incorporate these shifts, we note that (3.2) is equivalent to

$$
\widehat{X}+\sum_{r=1}^{m}\left(\widehat{K}_{r} \widehat{X} G_{r} \pm \alpha_{r} \widehat{X} G_{r}\right)=\widehat{\mathbf{f}}_{0} \mathbf{g}_{0}^{\top}
$$

which can be rearranged to give a matrix equation of the desired form (2.1),

$$
\widehat{X} \underbrace{\left(I-\sum_{r=1}^{m} \alpha_{r} G_{r}\right)}_{=: B_{0}}+\sum_{r=1}^{m} \underbrace{\left(\widehat{K}_{r}+\alpha_{r} I\right)}_{=: A_{r}} \hat{X} \underbrace{G_{r}}_{=: B_{r}}=\widehat{\mathbf{f}}_{0} \mathbf{g}_{0}^{\top} .
$$

The equivalent Kronecker formulation of (3.5) is given by $\mathcal{A} \widehat{\mathbf{x}}=\mathbf{b}$ with $\widehat{\mathbf{x}}=\operatorname{vec}(\widehat{X})$ and

$$
\mathcal{A}=B_{0} \otimes I+\sum_{r=1}^{m} B_{r} \otimes A_{r}, \quad \mathbf{b}=\mathbf{g}_{0} \otimes \widehat{\mathbf{f}}_{0} .
$$

In section 5 the efficiency of the low-rank projection method applied to (3.5) will be compared to that of solving the Kronecker formulation (3.6) using CG.

4. Implementation details. The complete low-rank projection algorithm consists of a preprocessing or set-up phase followed by an iterative solution phase. In the preprocessing phase, the matrices $K_{r}$ and $G_{r}$ are transformed into $A_{r}$ and $B_{r}$ and the vector $\mathbf{f}_{0}$ is transformed into $\widehat{\mathbf{f}}_{0}$, as outlined in the previous section. More precisely, the matrices $A_{r}$ and the vector $\widehat{\mathbf{f}}_{0}$ are not explicitly assembled. It is enough to provide the vector $\mathbf{f}_{0}$, the matrices $K_{r}$, the Cholesky factor $L$ of $K_{0}$, and the shifts $\alpha_{1}, \ldots, \alpha_{m}$. The parameters $\mathbf{s}=\left[s_{1}, s_{2}, \ldots\right]$ are also selected. Strategies for choosing them will be discussed in section 4.2. We stress that the preprocessing does not entail any approximation, and hence the transformed matrix equation (3.5) is mathematically equivalent to the original system (3.1). The solution phase is outlined in general terms in Algorithm 4.1. A careful implementation is essential if it is to be efficient in practice, and we address this next. 


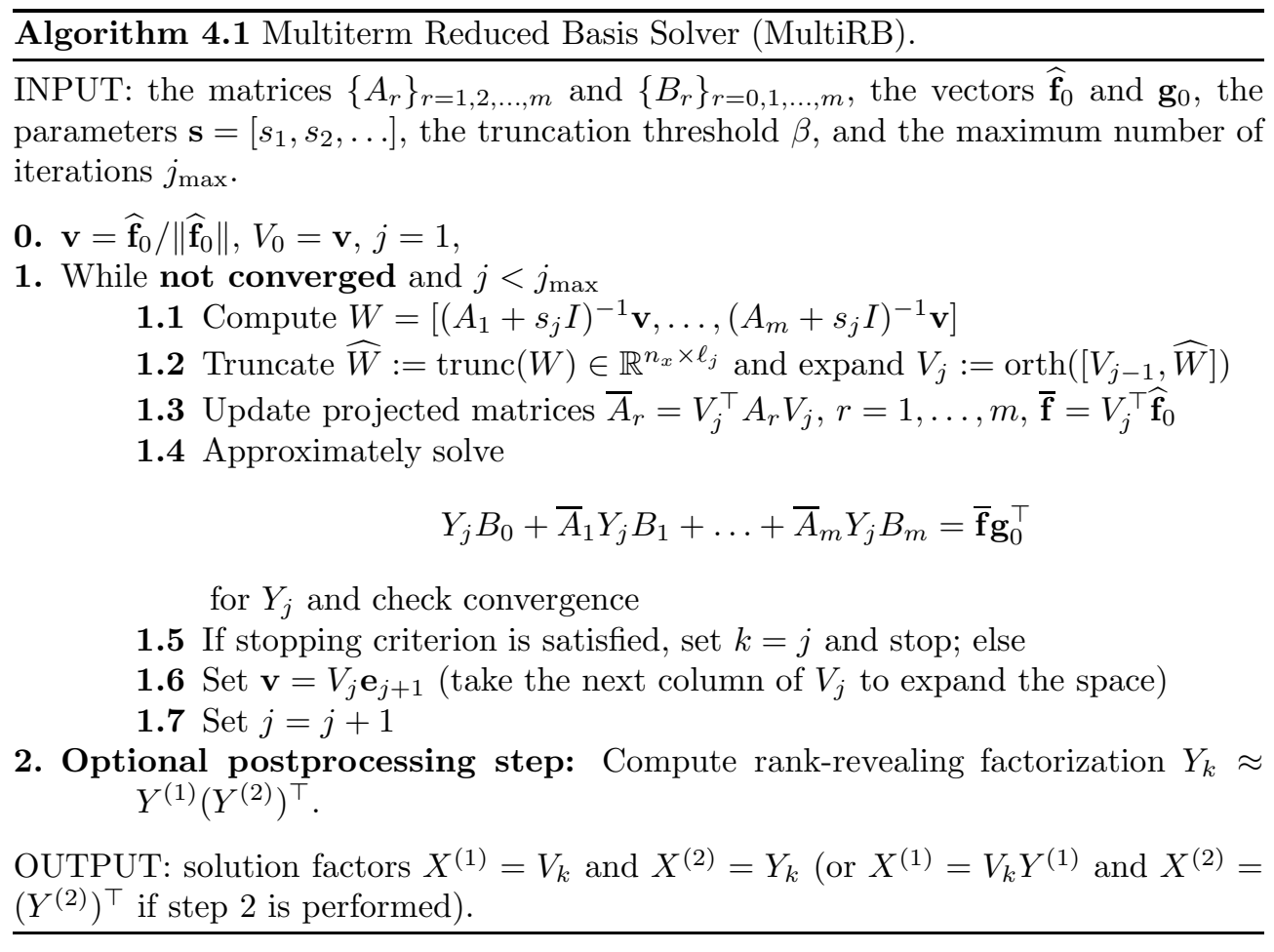

4.1. Solution phase. The most time-consuming parts of the solution phase are (i) step 1.1, which entails $m$ system solves with sparse matrices of dimension $n_{x} \times n_{x}$, (ii) step 1.2 , which performs the orthogonalization of the current reduced basis, and (iii) step 1.4, where the solution of the projected problem is computed and its accuracy is assessed. In step 1.2, the orthogonality of the approximation space basis is maintained by enforcing orthogonality of the newly added vectors by means of the Gram-Schmidt process. The new basis vectors are always taken to be the singular vectors corresponding to the dominant singular values of the latest computed matrix $W$. Note that steps 1.1 and 1.2 must be performed at every iteration, whereas step 1.4 can be performed every few iterations, if appropriate. We now elaborate on steps 1.1 and 1.4.

In step 1.1, the shifted systems that must be solved share the same right-hand side vector $\mathbf{v}$. In principle, they could be solved by a sparse direct solver. Indeed, if $s_{j}$ does not change with the iteration, and if memory is not a concern, then one could save sparse factorizations of each of the matrices $\left(A_{r}+s_{j} I\right)$ for $r=1, \ldots, m$ and reuse them in each iteration. For large $n_{x}$ and $m$, however, storing all the factorizations becomes prohibitive. Moreover, the direct solution of $m$ large systems may well be expensive. An effective alternative is the use of iterative methods and, in particular, the simultaneous iterative solution of all $m$ sparse systems using CG. The iterations for each system are performed at the same time. That is, the matrix-vector products are carried out simultaneously, making the access to memory allocations more efficient. It goes without saying that if iterative methods are used to solve the systems in step 1.1, then we do not need to solve them to machine precision. A loose tolerance will be employed. A crucial point is that the matrices $A_{r}=L^{-1} K_{r} L^{-\top}+\alpha_{r} I$ will never be explicitly assembled. If a system of the form $\left(A_{r}+s_{j} I\right) \mathbf{y}=\mathbf{v}$ is solved by a direct 
method, then this corresponds to solving

$$
\left(K_{r}+\left(\alpha_{r}+s_{j}\right) K_{0}\right) \widetilde{\mathbf{y}}=L \mathbf{v}, \quad \widetilde{\mathbf{y}}=L^{-\top} \mathbf{y},
$$

where $K_{r}$ and $K_{0}$ have the same sparsity pattern. On the other hand, if the same system is solved by an iterative method, then matrix-vector products of the type $\left(A_{r}+s_{j} I\right) \mathbf{w}$ can be performed as $\left(A_{r}+s_{j} I\right) \mathbf{w}=L^{-1}\left(\left(K_{r}+\left(\alpha_{r}+s_{j}\right) K_{0}\right)\left(L^{-\top} \mathbf{w}\right)\right)$. In either case operations with the sum $K_{r}+\left(\alpha_{r}+s_{j}\right) K_{0}$ are performed so $\widehat{K}_{r}$ is not formed explicitly.

In step 1.4, once the deterministic part of the problem has been reduced, the projected matrix equation needs to be solved and the quality of the current approximation assessed. We consider the Kronecker formulation and apply CG once more. As is typical in these circumstances, a matrix-oriented version of CG is employed to exploit the presence of blas 2 and blas 3 operations in all the required matrix operations. We also remark that to speed up convergence, the initial guess for this second CG iteration (step 1.4) is taken to be the solution $Y_{j-1}$ obtained at the previous outer iteration. To ensure the matrix has the correct dimension, we pad $Y_{j-1}$ with as many zero rows as basis vectors added to $V_{j}$ at step $j$. Summarizing, the algorithm contains two inner iterations: the first one expands the approximation space by solving $m$ linear systems with the same right-hand side, but with different coefficient matrices (step 1.1). The second one determines the approximate solution to the reduced problem (step 1.4). While for the former a fixed loose stopping tolerance can be used, for the latter an increasingly more accurate solution is sought as the outer iteration converges.

Monitoring convergence of the outer iteration is not straightforward. Since we assume that $n_{x}$ and $n_{\xi}$ are so large that vectors of dimension $n_{x} \cdot n_{\xi}$ should not be constructed, the residual matrix associated with the current approximation cannot be explicitly generated. Instead, at the $j$ th iteration we evaluate the relative difference

$$
\frac{\left\|X_{j}-X_{j-1}\right\|_{F}}{\left\|X_{j}\right\|_{F}}=\frac{\left\|Y_{j}-\left[Y_{j-1} ; 0\right]\right\|_{F}}{\left\|Y_{j}\right\|_{F}}
$$

where $\|\cdot\|_{F}$ denotes the Frobenius norm. For a matrix $X$, this norm is equivalent to computing the Euclidean norm of $\operatorname{vec}(X)$. The outer iteration is terminated once the relative difference in the approximation falls below a user-specified tolerance tol_outer. The stopping tolerance for the inner iteration in step 1.4 of Algorithm 4.1 can be tuned with respect to the outer tolerance. In section 5 we use a dynamically decreasing tolerance tol_inner for CG on the projected problems.

After $k$ iterations, when the outer iteration is judged to have converged, the approximate solution $X_{k}$ can be returned in factored form. That is, the matrices $X^{(1)}=V_{k}$ and $X^{(2)}=Y_{k}$ can be returned. However, the reduced solution matrix $Y_{k} \in \mathbb{R}^{n_{k} \times n_{\xi}}$ is not necessarily of full rank. Specifically, many of the singular values of $Y_{k}$ may fall below machine precision or some other tolerance that is below the final tolerance requested for convergence. This fact can be exploited at convergence in step 2. Details are given in the appendix. This computation may be expensive if $n_{k}$ and $n_{\xi}$ are both large so it may be skipped. It is not an essential part of the solution phase. However, the cost of performing step 2 is included in our experiments in section 5 . We also remark that the product $X^{(1)}\left(X^{(2)}\right)$ does not have to be performed explicitly. Single columns of the solution matrix can be computed as $X^{(1)}\left(\left(X^{(2)}\right) \mathbf{e}_{i}\right)$, $i=1, \ldots, n_{\xi}$, where $\mathbf{e}_{i}$ denotes the $i$ th column of the identity matrix. In particular, if we assume that the first basis function for $S_{p}$ is $\psi_{1}=1$, then the first column of the 
solution matrix provides the coefficients that determine the mean of the stochastic Galerkin approximation.

Finally, we note that the dimension of the approximation space constructed in Algorithm 4.1 is $n_{k}$ with $n_{k} \leq 1+m \cdot k$, since at most $m$ new vectors are added to the space at each iteration. Memory limitations may require the user to terminate the algorithm after a maximum number $j_{\max }$ of outer iterations - in this case the memory requirements are bounded by $\left(n_{x}+n_{\xi}\right) \cdot m \cdot j_{\max }$.

4.2. Selection of the parameters. It remains to choose the parameters $s_{j}$ used in step 1.1 of Algorithm 4.1. Recall that in section 2 we stipulated that $A_{1}, \ldots, A_{m}$ should all have eigenvalues contained in a small interval $\mathcal{S} \subset \mathbb{R}^{+}$. If this is true, then using a single parameter $s_{j}$ at the $j$ th iteration to construct all the columns of $W$ is justified. Two strategies for choosing $s_{j}$ are outlined below. To implement them, we need to estimate $\mathcal{S}$ cheaply, and so we explain how to do this first.

For stochastic Galerkin matrix equations we have $A_{r}=\widehat{K}_{r}+\alpha_{r} I, r=1, \ldots, m$, where the shift $\alpha_{r}$ is chosen to make $A_{r}$ positive definite. In section 3 we explained that $\alpha_{r}=1$ suffices and a bound for the spectral interval of $A_{r}$ is then given by $\mathcal{S}_{r}=\left[1-\tau_{r}, 1+\tau_{r}\right]$, where $\tau_{r}<1$ is defined in (3.3). Since the series (1.2) is assumed to converge as $m \rightarrow \infty$, we must also have $\tau_{r} \rightarrow 0$ as $r \rightarrow \infty$. Hence, the interval $\mathcal{S}_{r}$ contracts to $\alpha_{*}=1$ as $r \rightarrow \infty$ and $\mathcal{S}:=\mathcal{S}_{1}=\left[1-\tau_{1}, 1+\tau_{1}\right]$ contains the eigenvalues of each of $A_{1}, \ldots, A_{m}$. The end points of this interval can be estimated once the diffusion coefficient (1.2) has been chosen. No eigenvalues have to be computed.

It is often the case, especially when working with KL expansions (1.3), that the first eigenfunction $\phi_{1}$ is a strictly positive function, so that, as explained in section 3, the matrix $\widehat{K}_{1}$ is positive definite and $\mathcal{S}_{1}$ is not a sharp bound for the eigenvalues of $A_{1}=\widehat{K}_{1}+\alpha_{1} I$. However, for $r \geq 2$, the matrices $\widehat{K}_{r}$ are indefinite and do have spectral intervals centered around the origin (because the associated eigenfunctions are symmetric). For those matrices, the bound $\mathcal{S}_{r}$ is sharper. In this case, the following alternative strategy for choosing the shifts may be effective. Let $\lambda_{1, \min }, \lambda_{1, \max }$ be the extreme eigenvalues of $\widehat{K}_{1}$ and choose

$$
\alpha_{1}=1-\left(\lambda_{1, \min }+\lambda_{1, \max }\right) / 2, \quad \alpha_{2}=\alpha_{3}=\cdots=\alpha_{m}=1 .
$$

This ensures that the eigenvalues of $A_{r}=\widehat{K}_{r}+\alpha_{r} I$ for each $r=1, \ldots, m$ are centered around the unit value. The intervals $\mathcal{S}_{r}$ for $r \geq 2$ are now nested and collapse to the unit value as $r \rightarrow \infty$. Estimates for $\lambda_{1, \min }$ and $\lambda_{1, \max }$ can be obtained cheaply by computing the extreme eigenvalues of the matrix pencil $\left(K_{1}, K_{0}\right)$, where $K_{1}$ and $K_{0}$ are assembled on a coarse finite element mesh (since the eigenvalues do not depend on $h$ ). After applying the shifts in (4.3), the interval $\mathcal{S}:=\mathcal{S}_{2}$ or $\mathcal{S}=\left[1-\lambda_{2, \min }, 1+\lambda_{2, \max }\right]$, where $\lambda_{2, \min }$ and $\lambda_{2, \max }$ are estimates for the extreme eigenvalues of the matrix pencil $\left(K_{2}, K_{0}\right)$, typically contains the eigenvalues of each of $A_{1}, \ldots, A_{m}$. With an estimate of $\mathcal{S}$ in hand, we can now address how to choose the parameters $s_{j}$.

4.2.1. Multiple parameter strategy. One idea is to choose $s_{j}$ using standard parameter estimation ideas using rational approximation. For example, see [24]. Assume for simplicity that $m=1$, so that the problem to be solved is the Sylvester equation

$$
X B_{0}+A_{1} X B_{1}=\mathbf{f}_{0} \mathbf{g}_{0}^{\top} .
$$

Assume that $B_{0}$ is nonsingular and that we can write the full eigendecomposition $B_{1} B_{0}^{-1} Q=Q \Lambda$, where $\Lambda=\operatorname{diag}\left(\Lambda_{1}, 0\right)$ and $\Lambda_{1}$ is diagonal and nonsingular. Then 
(4.4) can be rewritten as

$$
X Q+A_{1} X Q \Lambda=\mathbf{f}_{0} \mathbf{g}_{0}^{\top} B_{0}^{-1} Q .
$$

Setting $Q=\left[Q_{1}, Q_{2}\right]$ with the same partitioning as for $\Lambda$, we see that the first block satisfies

$$
X Q_{1}+A_{1} X Q_{1} \Lambda_{1}=\mathbf{f}_{0} \mathbf{g}_{0}^{\top} B_{0}^{-1} Q_{1} .
$$

Moreover, the $j$ th column $\mathbf{z}_{j}$ of $X Q_{1}$ is the solution to a shifted system, $\left(\frac{1}{\lambda_{j}} I+A_{1}\right) \mathbf{z}_{j}=\mathbf{d}_{j}$, where $\mathbf{d}_{j}$ is the $j$ th column of the right-hand side matrix $\frac{1}{\lambda_{j}} \mathbf{f}_{0} \mathbf{g}_{0}^{\top} B_{0}^{-1} Q_{1}$. We now follow arguments for estimating $\mathbf{z}_{j}$ used in rational Galerkin methods. For example, see Druskin and Simoncini [6] and Druskin, Lieberman, and Zaslavsky [5]. An approximation of $\mathbf{z}_{j}$ is determined ${ }^{2}$ as $\mathbf{z}_{j, k} \in \overline{\mathbb{K}}_{k}\left(A_{1}, \mathbf{v}_{0}, \mathbf{s}\right)$, where

$$
\begin{aligned}
& \overline{\mathbb{K}}_{k}\left(A_{1}, \mathbf{v}_{0}, \mathbf{s}\right) \\
& =\operatorname{span}\left\{\left(A_{1}+s_{1} I\right)^{-1} \mathbf{v}_{0},\left(A_{1}+s_{2} I\right)^{-1}\left(A_{1}+s_{1} I\right)^{-1} \mathbf{v}_{0}, \ldots, \prod_{j=1}^{k}\left(A_{1}+s_{j} I\right)^{-1} \mathbf{v}_{0}\right\},
\end{aligned}
$$

and $\mathbf{v}_{0}$ is the normalized version of $\mathbf{d}_{j}$. The vector of parameters $\mathbf{s}$ is still to be selected. Let $\bar{A}_{1}=V_{k}^{\top} A_{1} V_{k}$, where the columns of $V_{k}$ are an orthonormal basis of $\overline{\mathbb{K}}_{k}\left(A_{1}, \mathbf{v}_{0}, \mathbf{s}\right)$. If a Galerkin condition is imposed to determine $\mathbf{z}_{j, k}$, then the residual satisfies (see [5])

$$
\mathbf{d}_{j}-\left(\frac{1}{\lambda_{j}} I+A_{1}\right) \mathbf{z}_{j, k}=\frac{r_{k}\left(A_{1}\right) \mathbf{d}_{j}}{r_{k}\left(-1 / \lambda_{j}\right)}, \quad r_{k}(x)=\prod_{j=1}^{k} \frac{x-\theta_{j}}{x+s_{j}},
$$

where $\theta_{j}$ are the eigenvalues of $\bar{A}_{1}$. We now have

$$
\min _{s_{1}, \ldots, s_{k}}\left\|\mathbf{d}_{j}-\left(\frac{1}{\lambda_{j}} I+A_{1}\right) \mathbf{z}_{j, k}\right\|=\min _{s_{1}, \ldots, s_{k}} \frac{1}{\left|r_{k}\left(-1 / \lambda_{j}\right)\right|}\left\|r_{k}\left(A_{1}\right) \mathbf{d}_{j}\right\|,
$$

where $\|\cdot\|$ denotes the Euclidean norm. We thus seek parameters $s_{1}, \ldots, s_{k}$ that minimize $\left\|r_{k}\left(A_{1}\right) \mathbf{d}_{j}\right\|$. A closely related optimization problem is obtained by replacing $\theta_{j}$ with $s_{j}$ in the numerator of $r_{k}$, giving the classical Zolotarev minimax problem

$$
\min _{s_{1}, \ldots, s_{k}} \max _{x \in \operatorname{spec}\left(A_{1}\right)}\left|\prod_{j=1}^{k} \frac{x-s_{j}}{x+s_{j}}\right| .
$$

If the Galerkin method is used with the values of $s_{j}$ obtained by solving this problem, then the rational function $r_{k}$ is the same as the function being minimized in the Zolotarev problem. That is, $\theta_{j}=s_{j}$ holds. To make the minimax problem tractable, $\operatorname{spec}\left(A_{1}\right)$ is usually replaced with $\mathcal{S}$, where $\mathcal{S}$ is an interval containing $\operatorname{spec}\left(A_{1}\right)$. The set of parameters solving this problem can be computed using elliptic functions. Their derivation is discussed, e.g., by Lu and Wachspress [16], and their computation by a few lines of MATLAB code is described by Sabino [22, p. 43]. Other selections associated with quasi-optimal rational approximation of matrix functions could be used

\footnotetext{
${ }^{2}$ To simplify the derivation, $\mathbf{v}_{0}$ is not included in the approximation space.
} 
instead; see, for example, Beckermann and Reichel [3] or Güttel [12], and Simoncini [24] for a general presentation in the context of linear matrix equations.

In our setting (with $m>1$ ), given an estimate for the interval $\mathcal{S}$ that contains the eigenvalues of each of the matrices $A_{1}, \ldots, A_{m}$, we may compute, say, 5 or 10 nodes $s_{j} \in \mathcal{S}$ using the method described above, which we cycle through as the iteration proceeds.

4.2.2. Parameter-free strategy. Looking at (4.1) we can see that both $\alpha_{r}$ and $s_{j}$ act as shifts, although the rationale presented for choosing them is different. In the case of stochastic Galerkin matrix equations, the shifts $\alpha_{r}$ and the parameters $s_{j}$ can often be combined, leading to a simpler version of Algorithm 4.1. We now develop this idea.

Instead of selecting, say, 5 or 10 parameters $s_{j} \in \mathcal{S}$ as described above, a simpler strategy is to choose $s_{j}=s_{*}$ for each $j=1,2, \ldots$ That is, choose a single point in $\mathcal{S}$ and use the same parameter in each iteration. For Lyapunov equations, the choice $s_{*}=\sqrt{a \cdot b}$ was suggested in [16], where the estimated spectral interval is given by $\mathcal{S}=[a, b]$. In our setting, if we choose the shifts to be $\alpha_{r}=1$ for $r=1, \ldots, m$, then each of the intervals $\mathcal{S}_{r}$ will be centered about $\alpha_{*}=1$, and a natural choice is $s_{*}=1$ (the mid-point). $\mathcal{S}$ does not even need to be estimated. In (4.1), we then have $\alpha_{r}+s_{j}=2$ for $r=1, \ldots, m$, in each iteration. A mathematically equivalent version of Algorithm 4.1 is then obtained by setting the constants $\alpha_{r}$ to zero and choosing $s_{*}=\left(\alpha_{r}+s_{j}\right)=2$. Note that with this combination, the preprocessing phase is essentially redundant. Only the Cholesky factor of $K_{0}$ needs to be precomputed. Once the truncation threshold $\beta$ is fixed, the MultiRB solver is completely parameter-free!

Whether we use a multiparameter or parameter-free strategy, it is worth noting that if the spectrum of each matrix $A_{r}, r=1, \ldots, m$, is independent of the mesh parameter $h$, as in our setting, then the computed values of $s_{j}$ do not depend on $h$. Since the spectrum of each matrix $A_{r}+s_{j} I$ is then independent of the mesh parameter, we anticipate that the dimension of the approximation space generated by our algorithm will be insensitive to $h$. This feature will be confirmed by numerical experiments next.

5. Numerical experiments. In this final section, we consider two test problems of the form (1.1) that are implemented in the MATLAB software package S-IFISS [23]. The iterative solver in S-IFISS tackles the Kronecker formulation of the original linear system $\mathcal{A} \mathbf{x}=\mathbf{b}$ with $\mathcal{A}$ in (1.7), not the matrix equation formulation considered here. Example 5.1 corresponds to test problem 5 in S-IFISS and has a diffusion coefficient of the form (1.2) taken from Eigel et al. [7]. Example 5.2 corresponds to S-IFISS test problem 2, and in this case, the diffusion coefficient is a truncated KL expansion (1.3) with relatively slowly decaying eigenvalues $\lambda_{r}$. In both examples, we choose the space $Z_{h}$ to be the set of piecewise bilinear functions defined on uniform subdivisions of a square domain $D$. We perform experiments with two specific finite element grids with $n_{x}=16,129$ and $n_{x}=65,025$ degrees of freedom. The second of these (grid level 8) is a uniform refinement of the first (grid level 7). We choose the space $S_{p}$ to be the set of Legendre polynomials of total degree $p$ in $y_{1}, \ldots, y_{m}$ on $\Gamma=[-1,1]^{m}$. We vary $p$ and choose $m$ appropriately for the problem at hand. Recall that $n_{\xi}$ is given by (1.6). All experiments were performed in MATLAB 7.10.0 on a Dell Precision T7500 desktop computer with 12 cores and a total of 48 GB RAM.

To solve the test problems we apply (i) the new MultiRB solver (Algorithm 4.1) to the matrix equation (3.5) and (ii) CG to the equivalent Kronecker formulation (3.6). Notice that both methods essentially use the mean-based preconditioner $P=I \otimes K_{0}$. 
We compare the efficiency of the two approaches and investigate how the dimension $n_{k}$ of the approximation space constructed by the MultiRB solver behaves when we increase the number of spatial degrees of freedom $n_{x}$, the polynomial degree $p$, and the number of random variables $m$. All timings reported are in seconds. In the implementation of MultiRB, we apply CG in step 1.1 until the relative residual error for all $m$ systems is below $10^{-4}$. The stopping tolerance for the outer iteration is chosen to be tol_outer $=10^{-5}$. The projected problem in step 1.4 is solved iteratively with CG at each iteration, and we use the dynamically decreasing inner tolerance tol_inner $=10^{-3}$. tol_outer. Unless otherwise stated, we fix the truncation threshold to be $\beta=99$.

When we apply CG to the Kronecker formulation, we exploit the structure and use (1.9) when performing matrix-vector products. Variants of CG for linear systems in Kronecker form are written with matrix-matrix operations and typically include truncation strategies that maintain iterates of low rank as the iteration proceeds; see, for example [18]. We do not adopt such a truncation strategy in our comparison because doing so would affect the optimality properties of the CG solver and we want our reference method to have a reliable convergence rate. To make fair comparisons with the reduced basis solver, the stopping criterion for CG applied to (3.6) is also based on the relative difference in the approximate solution. The memory requirements of CG applied to (3.6) are 4 vectors of length $n_{x} \cdot n_{\xi}$ each: the current approximation, the direction, the residual, and an extra vector for storing the matrix-vector multiplication; the total memory requirements are thus $4 \cdot n_{x} \cdot n_{\xi}$. In contrast, the memory requirements for the reduced basis solver are of order $\left(n_{x}+n_{\xi}\right) \cdot n_{k}$. We stress that CG requires full length vectors of size $n_{x} \cdot n_{\xi}$ whereas the new solver does not.

Example 5.1. Consider the square domain $D=[0,1] \times[0,1]$ and define the diffusion coefficient to be (1.2) with $a_{0}=1$ and $a_{r}(\vec{x})=\gamma_{r} \cos \left(2 \pi \beta_{1}(r) x_{1}\right) \cos \left(2 \pi \beta_{2}(r) x_{2}\right)$, where $\gamma_{r}:=0.832 r^{-4}$ and $\beta_{1}(r):=r-s(r)(s(r+1)) / 2, \beta_{2}(r):=s(r)-\beta_{1}(r)$ with $s(r):=\lfloor-1 / 2+\sqrt{1 / 4+2 r}\rfloor$. We note that the coefficients $\gamma_{r}$, which determine the weights of the terms $a_{r}$, decay rapidly as $r \rightarrow \infty$. If we choose $m=5$, then we retain all terms in (1.2) with coefficients $\gamma_{r} \geq 10^{-3}$. However, if $m=9$ and $m=16$, then we retain all terms with $\gamma_{r} \geq 10^{-4}$ and $10^{-5}$, respectively. Selected polynomial chaos coefficients $u_{j}$ of the computed approximation

$$
u_{h p}(\vec{x}, \mathbf{y})=\sum_{j=1}^{n_{\xi}} u_{j}(\vec{x}) \psi_{j}(\mathbf{y})
$$

are shown in Figure 1 for the case $m=9$ and $p=3$. Note that $u_{1}$ represents the mean $\mathbb{E}\left[u_{h p}\right]$.

First, we fix the grid level to be 7 and compare the multiple-parameter and parameter-free implementations of the reduced basis solver outlined in sections 4.2.1 and 4.2.2. For the latter, we set $s_{*}=2$. For the former, we choose the shifts as in (4.3) and then, on the estimated interval $\mathcal{S}=\left[\alpha_{1}+\lambda_{1, \min }, \alpha_{1}+\lambda_{1, \max }\right] \approx[0.1695,1.8305]$, we compute 5 nodes $\left[s_{1}, s_{2}, s_{3}, s_{4}, s_{5}\right]$ using the MATLAB code from [22, p. 43]. Note that $\alpha_{1}$ is close to 1 in this example since $\lambda_{1, \min } \approx-\lambda_{1, \max }$. The matrix $\widehat{K}_{1}$ is indefinite. Results are presented in Table 1 . We record the number of outer iterations $k$ required by the MultiRB solver, the dimension $n_{k}$ of the approximation space generated (the number of columns of $V_{k}$ ), and the rank of the final approximation $X_{k}$. We also record the CPU time in seconds for the solution phase. This includes the time taken to compute the truncated singular value decomposition of $Y_{k}$. For $m=9$ and $m=16$ we 

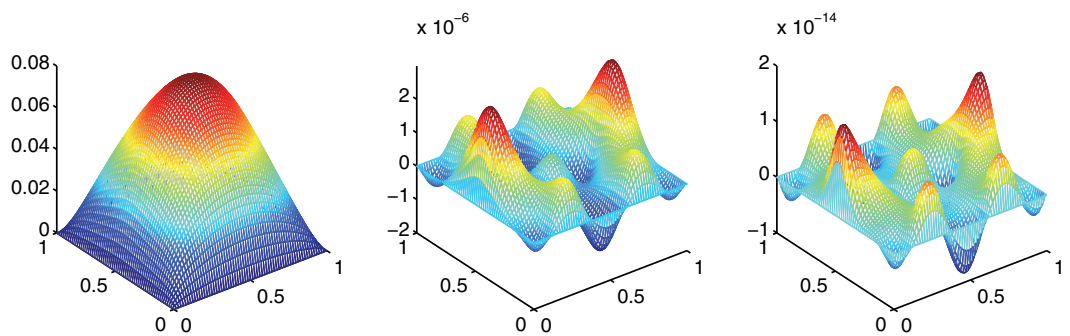

Fig. 1. Left to right: Polynomial chaos coefficients $u_{1}, u_{5}$, and $u_{10}$ of the stochastic Galerkin solution $u_{h p} \in Z_{h} \otimes S_{p}$ to Example 5.1 in the case $m=9$ and $p=3$. The left plot shows the mean solution.

TABLE 1

Results obtained with the MultiRB solver for Example 5.1 and grid level 7: multiparameter versus parameter-free.

\begin{tabular}{|c|rr|rrrr||rrrr|}
\hline \multicolumn{9}{|c|}{} & & \multicolumn{5}{|c||}{ Multiparameter } & \multicolumn{4}{c|}{ Parameter-free } \\
\hline$m$ & $p$ & $n_{\xi}$ & $k$ & $n_{k}$ & Rank & Time & $k$ & $n_{k}$ & Rank & Time \\
\hline \multirow{3}{*}{5} & 2 & 21 & 23 & 84 & 19 & $5.96 \mathrm{e} 0$ & 16 & 66 & 19 & $3.67 \mathrm{e} 0$ \\
& 3 & 56 & 26 & 97 & 29 & $7.02 \mathrm{e} 0$ & 19 & 77 & 28 & $4.76 \mathrm{e} 0$ \\
& 4 & 126 & 28 & 104 & 37 & $7.80 \mathrm{e} 0$ & 19 & 77 & 36 & $4.63 \mathrm{e} 0$ \\
& 5 & 252 & 28 & 104 & 43 & $8.24 \mathrm{e} 0$ & 23 & 94 & 42 & $6.29 \mathrm{e} 0$ \\
\hline \multirow{3}{*}{9} & 2 & 55 & 16 & 74 & 26 & $6.96 \mathrm{e} 0$ & 14 & 79 & 26 & $5.72 \mathrm{e} 0$ \\
& 3 & 220 & 16 & 74 & 34 & $7.21 \mathrm{e} 0$ & 16 & 94 & 34 & $6.96 \mathrm{e} 0$ \\
& 4 & 715 & 16 & 74 & 40 & $7.84 \mathrm{e} 0$ & 17 & 100 & 42 & $8.19 \mathrm{e} 0$ \\
& 5 & 2,002 & 22 & 105 & 47 & $1.67 \mathrm{e} 1$ & 18 & 102 & 47 & $1.27 \mathrm{e} 1$ \\
\hline \multirow{5}{*}{16} & 2 & 153 & 15 & 87 & 32 & $1.19 \mathrm{e} 1$ & 12 & 82 & 32 & $9.94 \mathrm{e} 0$ \\
& 3 & 969 & 16 & 89 & 40 & $1.40 \mathrm{e} 1$ & 14 & 106 & 41 & $1.21 \mathrm{e} 1$ \\
& 4 & 4,845 & 16 & 89 & 45 & $2.53 \mathrm{e} 1$ & 15 & 117 & 46 & $2.82 \mathrm{e} 1$ \\
& 5 & 20,349 & 19 & 105 & 49 & $1.71 \mathrm{e} 2$ & 15 & 117 & 51 & $1.52 \mathrm{e} 2$ \\
\hline
\end{tabular}

TABLE 2

Results for Example 5.1 and grid level 7: MultiRB solver versus standard CG.

\begin{tabular}{|c|cr|cc|ccc||r|}
\hline$m$ & $p$ & $n_{\xi}$ & $k$ & Inner & $n_{k}$ & Rank & Time & CG time (its) \\
\hline \multirow{5}{*}{5} & 2 & 21 & 16 & 12.9 & 66 & 19 & $3.67 \mathrm{e} 0$ & $1.42 \mathrm{e} 0(10)$ \\
& 3 & 56 & 19 & 15.6 & 77 & 28 & $4.76 \mathrm{e} 0$ & $3.85 \mathrm{e} 0(12)$ \\
& 4 & 126 & 19 & 16.7 & 77 & 36 & $4.63 \mathrm{e} 0$ & $9.32 \mathrm{e} 0(14)$ \\
& 5 & 252 & 23 & 18.9 & 94 & 42 & $6.29 \mathrm{e} 0$ & $1.77 \mathrm{e} 1(14)$ \\
\hline \multirow{3}{*}{9} & 2 & 55 & 14 & 13.3 & 79 & 26 & $5.72 \mathrm{e} 0$ & $3.68 \mathrm{e} 0(10)$ \\
& 3 & 220 & 16 & 15.6 & 94 & 34 & $6.96 \mathrm{e} 0$ & $1.59 \mathrm{e} 1(12)$ \\
& 4 & 715 & 17 & 17.1 & 100 & 42 & $8.19 \mathrm{e} 0$ & $6.20 \mathrm{e} 1(14)$ \\
& 5 & 2,002 & 18 & 18.4 & 102 & 47 & $1.27 \mathrm{e} 1$ & $1.71 \mathrm{e} 2(14)$ \\
\hline \multirow{3}{*}{16} & 2 & 153 & 12 & 12.7 & 82 & 32 & $8.94 \mathrm{e} 0$ & $1.26 \mathrm{e} 1(10)$ \\
& 3 & 969 & 14 & 15.1 & 106 & 41 & $1.21 \mathrm{e} 1$ & $1.64 \mathrm{e} 1(12)$ \\
& 4 & 4,845 & 15 & 16.7 & 117 & 46 & $2.82 \mathrm{e} 1$ & $5.44 \mathrm{e} 2(14)$ \\
& 5 & 20,349 & 15 & 17.5 & 117 & 51 & $1.52 \mathrm{e} 2$ & $3.31 \mathrm{e} 3(14)$ \\
\hline
\end{tabular}

see that the approximation space generated by the multiparameter version is smaller than that generated by the parameter-free version. However, timings are similar for both implementations.

Adopting the parameter-free implementation for simplicity, we now compare the efficiency of using the MultiRB method to solve the matrix equation (3.5) and using CG to solve (3.6). Results obtained for grid levels 7 and 8 are presented in Tables 2 
TABLE 3

Results for Example 5.1 and grid level 8: MultiRB solver versus standard CG.

\begin{tabular}{|c|rr|cc|ccc||r|}
\hline$m$ & $p$ & $n_{\xi}$ & $k$ & Inner & $n_{k}$ & Rank & Time & CG time (its) \\
\hline \multirow{3}{*}{5} & 2 & 21 & 16 & 12.9 & 66 & 19 & $2.14 \mathrm{e} 1$ & $8.13 \mathrm{e} 0(10)$ \\
& 3 & 56 & 19 & 15.6 & 77 & 28 & $2.65 \mathrm{e} 1$ & $2.17 \mathrm{e} 1(12)$ \\
& 4 & 126 & 19 & 16.7 & 77 & 36 & $2.52 \mathrm{e} 1$ & $5.31 \mathrm{e} 1(14)$ \\
& 5 & 252 & 23 & 18.9 & 94 & 42 & $3.23 \mathrm{e} 1$ & $1.03 \mathrm{e} 2(14)$ \\
\hline \multirow{3}{*}{9} & 2 & 55 & 14 & 13.3 & 79 & 26 & $3.27 \mathrm{e} 1$ & $2.03 \mathrm{e} 1(10)$ \\
& 3 & 220 & 16 & 15.6 & 94 & 34 & $3.86 \mathrm{e} 1$ & $9.11 \mathrm{e} 1(12)$ \\
& 4 & 715 & 17 & 17.1 & 100 & 42 & $4.22 \mathrm{e} 1$ & $3.35 \mathrm{e} 2(14)$ \\
& 5 & 2,002 & 18 & 18.3 & 102 & 47 & $4.91 \mathrm{e} 1$ & $9.35 \mathrm{e} 2(14)$ \\
\hline \multirow{3}{*}{16} & 2 & 153 & 12 & 12.7 & 82 & 32 & $5.04 \mathrm{e} 1$ & $6.76 \mathrm{e} 1(10)$ \\
& 3 & 969 & 14 & 15.1 & 106 & 41 & $6.19 \mathrm{e} 1$ & $4.90 \mathrm{e} 2(12)$ \\
& 4 & 4,845 & 15 & 16.7 & 117 & 46 & $8.47 \mathrm{e} 1$ & $2.81 \mathrm{e} 3(14)$ \\
& 5 & 20,349 & 15 & 17.5 & 117 & 51 & $2.15 \mathrm{e} 2$ & Out of Memory \\
\hline
\end{tabular}

and 3. In column five, we also now record the average number of inner CG iterations required to solve the projected matrix equations. The CPU time in seconds taken to solve the Kronecker formulation using CG is reported in the final column, and the associated number of CG iterations is given in parentheses for reference.

Comparing the results in Tables 2 and 3 we see that the dimension $n_{k}$ of the approximation space built by the MultiRB solver is independent of $h$, as predicted. The rank of the final approximation is also observed to be independent of $h$. When $n_{\xi}$ is small we see that there is nothing to be gained by using the low-rank solver. Using $\mathrm{CG}$ on the Kronecker formulation is quicker and requires comparable memory. This can be explained as follows. To construct the reduced basis of size $n_{k}$, the new method requires the solution of $m \cdot k$ linear systems of size $n_{x}$. In each iteration, mean-based preconditioned CG requires the solution of $n_{\xi}$ linear systems of size $n_{x}$ (with coefficient matrix $K_{0}$ ). So, when $n_{\xi} \ll m \cdot k$, CG performs fewer solves and is quicker overall. However, when the choice of $m$ and $p$ is such that the dimension $n_{\xi}$ is large (typically $n_{\xi}>100$ ), using the reduced basis solver on the matrix formulation is much quicker. Although we do not report them here, we note that the results obtained with the multiparameter implementation of the low-rank solver exhibit completely analogous behavior. Of course, timings are not the only consideration. In the largest experiment, where $n_{\xi}=20,349$ and the grid level is 8 , the Kronecker formulation consists of 1.3 billion equations! We run out of memory when we apply CG to (3.6). However, since the reduced basis solver never forms vectors of length $n_{x} \cdot n_{\xi}$, it is able to make more efficient use of the available memory. As mentioned in section 4.2, when $n_{\xi}$ and $n_{x}$ are large, computing the truncated singular value decomposition of $Y_{k}$ is costly. In Table 3 , in the case $m=16$ and $p=5$, we note that $35 \%$ of the total reported solution time for the reduced basis solver was spent on this postprocessing task. Recall that this step is not part of the actual solution phase and can be turned off by the user.

We observe that both the dimension $n_{k}$ of the approximation space generated by the reduced basis solver and the rank of the final solution matrix increase slightly with the number of random variables $m$ and the polynomial degree $p$. Notice also that $n_{k}$ is larger than the rank in all cases. This suggests that the reduced basis built by the new method is not optimal. One possible reason for this is that since the terms in (1.2) decay so rapidly in this example, the spectral intervals of the matrices $A_{r}$ also decay very rapidly. The matrix $A_{1}$ has a noticeably larger spectral interval than $A_{2}, \ldots, A_{m}$. Choosing a parameter $s_{j}$ to form $\left(A_{1}+s_{j} I\right)$ that is distinct from the 
TABLE 4

Results for Example 5.1 and grid level 8: MultiRB solver with $\beta=96$.

\begin{tabular}{|c|rr|cc|rrr|}
\hline$m$ & $p$ & $n_{\xi}$ & $k$ & Inner & $n_{k}$ & Rank & Time \\
\hline \multirow{3}{*}{9} & 2 & 55 & 23 & 13.1 & 82 & 26 & $4.94 \mathrm{e} 1$ \\
& 3 & 220 & 24 & 14.8 & 83 & 34 & $5.10 \mathrm{e} 1$ \\
& 4 & 715 & 27 & 16.8 & 93 & 42 & $5.97 \mathrm{e} 1$ \\
& 5 & 2,002 & 27 & 17.6 & 95 & 47 & $6.44 \mathrm{e} 1$ \\
\hline
\end{tabular}
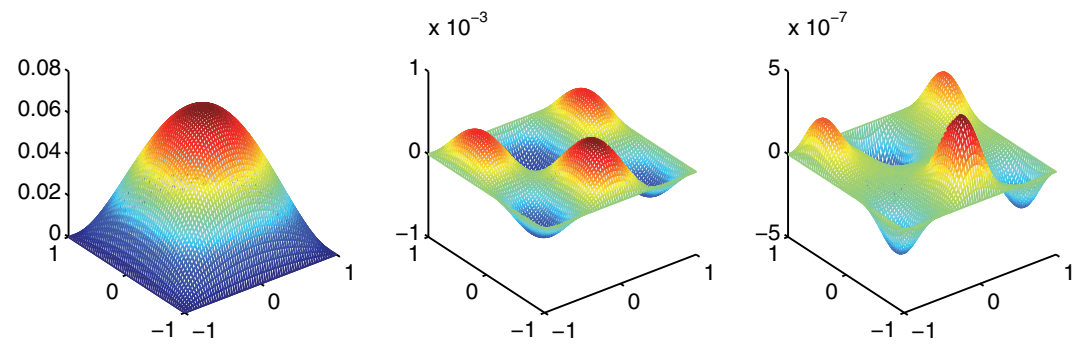

Fig. 2. Left to right: Polynomial chaos coefficients $u_{1}, u_{5}$ and $u_{10}$ of the stochastic Galerkin solution $u_{h p} \in Z_{h} \otimes S_{p}$ to Example 5.2 in the case $m=12, p=3$ and $\sigma=0.3$. The left plot shows the mean solution.

parameter $s_{j}$ used to form $\left(A_{r}+s_{j} I\right)$ for $r \geq 2$ in the construction of $W$ in step 1.1 of the MultiRB method may then be more effective. The current implementation is more general, however, and the savings in both time and memory usage compared to using standard CG are self-evident.

In the results above we used the default value $\beta=99$ for the truncation threshold. The results presented in Table 4 illustrate the effect of relaxing the threshold to $\beta=96$ so that fewer vectors are retained at each step. Comparing the results in Table 4 with those in Table 3 for the case $m=9$ we see that although the dimension $n_{k}$ is now a little smaller, more outer iterations are required and the timings have increased.

Example 5.2. Consider the domain $D=[-1,1] \times[-1,1]$ and let the diffusion coefficient now take the form (1.3) with mean $\mu=1$. Let $\xi_{r} \sim U(-\sqrt{3}, \sqrt{3})$ and consider the covariance function

$$
C\left(\vec{x}_{1}, \vec{x}_{2}\right)=\sigma^{2} \exp \left(-\frac{\left\|\vec{x}_{1}-\vec{x}_{2}\right\|_{1}}{\ell}\right),
$$

where $\ell$ denotes the correlation length. The eigenvalues and eigenfunctions of the associated covariance operator can be computed analytically (e.g., see [15, Chapter 7]). The eigenvalues $\lambda_{r}$ determine the weightings of the individual terms in the expansion (1.3). Note that these decay much more slowly than the coefficients $\gamma_{r}$ in Example 5.1. If we set $m=\infty$ in $(1.3)$, then $\int_{D} \operatorname{Var}(a(\vec{x}, \omega)) d \vec{x}=\sigma^{2} \operatorname{Leb}(D)=4 \sigma^{2}$. If we truncate the series after $m$ terms, then the integral of the variance of the approximated coefficient is $\sigma^{2} \sum_{r=1}^{m} \lambda_{r}$. We can use this fact to determine how to choose $m$. In particular, setting $\ell=2$ and then retaining $m=8,12$, and 20 terms means that we incorporate $87 \%, 89 \%$, and $93 \%$, respectively, of the integral of the variance of $a$. We present results below for $\ell=2$ and two distinct values of the standard deviation: $\sigma=0.1$ and $\sigma=0.3$. Selected polynomial chaos coefficients $u_{j}$ of the computed approximation are shown in Figure 2 for the case $m=12, p=3$, and $\sigma=0.3$. 
TABLE 5

Results obtained with the MultiRB solver for Example 5.2 with $\sigma=0.1$ and grid level 7: multiparameter versus parameter-free implementations.

\begin{tabular}{|c|rr|rrrr||rrrr|}
\hline \multicolumn{3}{|c|}{} & \multicolumn{4}{c||}{ Multiparameter } & \multicolumn{4}{c|}{ Parameter-free } \\
\hline$m$ & $p$ & $n_{\xi}$ & $k$ & $n_{k}$ & Rank & Time & $k$ & $n_{k}$ & Rank & Time \\
\hline \multirow{3}{*}{8} & 2 & 45 & 8 & 58 & 40 & $2.27 \mathrm{e} 0$ & 8 & 59 & 40 & $2.18 \mathrm{e} 0$ \\
& 3 & 165 & 9 & 66 & 65 & $2.50 \mathrm{e} 0$ & 9 & 67 & 66 & $2.71 \mathrm{e} 0$ \\
& 4 & 495 & 9 & 66 & 66 & $2.65 \mathrm{e} 0$ & 9 & 67 & 66 & $2.75 \mathrm{e} 0$ \\
& 5 & 1,287 & 9 & 66 & 66 & $3.01 \mathrm{e} 0$ & 9 & 67 & 66 & $3.08 \mathrm{e} 0$ \\
\hline \multirow{3}{*}{12} & 2 & 91 & 7 & 76 & 62 & $3.25 \mathrm{e} 0$ & 8 & 87 & 63 & $3.86 \mathrm{e} 0$ \\
& 3 & 455 & 8 & 87 & 87 & $3.98 \mathrm{e} 0$ & 8 & 87 & 87 & $3.99 \mathrm{e} 0$ \\
& 4 & 1,820 & 8 & 87 & 87 & $5.07 \mathrm{e} 0$ & 8 & 87 & 87 & $5.10 \mathrm{e} 0$ \\
& 5 & 6,188 & 8 & 87 & 87 & $1.23 \mathrm{e} 1$ & 8 & 87 & 87 & $1.22 \mathrm{e} 1$ \\
\hline \multirow{3}{*}{20} & 2 & 231 & 10 & 172 & 125 & $1.07 \mathrm{e} 1$ & 10 & 171 & 125 & $1.21 \mathrm{e} 1$ \\
& 3 & 1,771 & 10 & 172 & 172 & $1.54 \mathrm{e} 1$ & 10 & 171 & 171 & $1.41 \mathrm{e} 1$ \\
& 4 & 10,626 & 10 & 172 & 172 & $5.38 \mathrm{e} 1$ & 10 & 171 & 171 & $5.12 \mathrm{e} 1$ \\
\hline
\end{tabular}

TABLE 6

Results obtained with the MultiRB solver for Example 5.2 with $\sigma=0.3$ and grid level 7: multiparameter versus parameter-free implementations.

\begin{tabular}{|c|rr|rrrr||rrrr|}
\hline \multicolumn{2}{|c|}{} & \multicolumn{4}{c||}{ Multiparameter } & \multicolumn{4}{c|}{ Parameter-free } \\
\hline$m$ & $p$ & $n_{\xi}$ & $k$ & $n_{k}$ & Rank & Time & $k$ & $n_{k}$ & Rank & Time \\
\hline \multirow{3}{*}{8} & 2 & 45 & 16 & 124 & 45 & $5.37 \mathrm{e} 0$ & 17 & 128 & 45 & $5.57 \mathrm{e} 0$ \\
& 3 & 165 & 21 & 163 & 128 & $8.08 \mathrm{e} 0$ & 20 & 152 & 127 & $7.18 \mathrm{e} 0$ \\
& 4 & 495 & 24 & 187 & 182 & $1.05 \mathrm{e} 1$ & 24 & 183 & 178 & $1.04 \mathrm{e} 1$ \\
& 5 & 1,287 & 26 & 203 & 203 & $1.87 \mathrm{e} 1$ & 27 & 207 & 207 & $1.87 \mathrm{e} 1$ \\
\hline \multirow{3}{*}{12} & 2 & 91 & 15 & 166 & 89 & $8.52 \mathrm{e} 0$ & 15 & 165 & 89 & $8.54 \mathrm{e} 0$ \\
& 3 & 455 & 18 & 202 & 197 & $1.22 \mathrm{e} 0$ & 18 & 201 & 197 & $1.20 \mathrm{e} 1$ \\
& 4 & 1,820 & 21 & 236 & 236 & $2.59 \mathrm{e} 1$ & 21 & 236 & 236 & $2.60 \mathrm{e} 1$ \\
& 5 & 6,188 & 25 & 282 & 282 & $1.17 \mathrm{e} 2$ & 25 & 282 & 282 & $1.14 \mathrm{e} 2$ \\
\hline \multirow{3}{*}{20} & 2 & 231 & 16 & 283 & 203 & $2.08 \mathrm{e} 1$ & 16 & 281 & 206 & $2.00 \mathrm{e} 1$ \\
& 3 & 1,771 & 23 & 403 & 403 & $6.45 \mathrm{e} 1$ & 23 & 399 & 399 & $6.28 \mathrm{e} 1$ \\
& 4 & 10,626 & 26 & 459 & 459 & $4.38 \mathrm{e} 2$ & 26 & 454 & 454 & $4.11 \mathrm{e} 2$ \\
\hline
\end{tabular}

First, we fix the grid level to be 7 and compare the multiple-parameter and parameter-free implementations of the reduced basis solver. For the latter, we set $s_{*}=2$ as before. For the former, we choose the shifts as in (4.3). The matrix $\widehat{K}_{1}$ is positive definite now. In the case $\sigma=0.1$ (the low standard deviation case), we obtain $\alpha_{1}=0.8794$. The eigenvalues of $A_{1}$ are then contained in $[0.9737,1.0263]$. This interval is not large enough to contain the eigenvalues of each of $A_{2}, \ldots, A_{m}$ so we use $\mathcal{S}=\left[1+\lambda_{2, \min }, 1+\lambda_{2, \max }\right] \approx[0.9219,1.0784]$. Similarly, in the case $\sigma=0.3$, we obtain $\alpha_{1}=0.6381$ and use $\mathcal{S}=\left[1+\lambda_{2, \min }, 1+\lambda_{2, \max }\right] \approx[0.7657,1.2344]$. Notice that $\mathcal{S}$ is larger when $\sigma$ increases. For both values of the standard deviation, we compute 5 nodes $\left[s_{1}, s_{2}, s_{3}, s_{4}, s_{5}\right]$ on $\mathcal{S}$, using the MATLAB code from [22, p. 43] as before. Results are presented in Tables 5 and 6 .

We see that both implementations of the reduced basis solver work equally well. This is no surprise since the end points of the interval $\mathcal{S}$ used by the multiparameter version are a much smaller perturbation from 1 here than in Example 5.1. The computed values of $s_{j}$ are all close to 1 (the midpoint of $\mathcal{S}$ ), especially when $\sigma=0.1$, which is effectively the choice made in the parameter-free version. It is also interesting to note that both versions of our new method generate approximation spaces whose dimensions match the rank of the final approximation (unless $n_{\xi}$ is small). In this sense, the reduced basis is optimal. Notice also that the recorded ranks are much larger than in Example 5.1 and they increase when both the standard deviation $\sigma$ and the 
TABLE 7

Results for Example 5.2 with $\sigma=0.3$ and grid level 7: MultiRB solver versus standard $C G$.

\begin{tabular}{|c|rr|rr|rrr||c|}
\hline$m$ & $p$ & $n_{\xi}$ & $k$ & Inner & $n_{k}$ & Rank & Time & CG time (its) \\
\hline \multirow{3}{*}{8} & 2 & 45 & 17 & 9.8 & 128 & 45 & $5.57 \mathrm{e} 0$ & $2.47 \mathrm{e} 0(8)$ \\
& 3 & 165 & 20 & 12.1 & 152 & 127 & $7.18 \mathrm{e} 0$ & $9.93 \mathrm{e} 0(10)$ \\
& 4 & 495 & 24 & 14.5 & 183 & 178 & $1.04 \mathrm{e} 1$ & $3.58 \mathrm{e} 1(12)$ \\
& 5 & 1,287 & 27 & 17.0 & 207 & 207 & $1.87 \mathrm{e} 1$ & $9.90 \mathrm{e} 1(13)$ \\
\hline \multirow{3}{*}{12} & 2 & 91 & 15 & 9.9 & 165 & 89 & $8.54 \mathrm{e} 0$ & $5.52 \mathrm{e} 0(8)$ \\
& 3 & 455 & 18 & 12.2 & 201 & 197 & $1.20 \mathrm{e} 1$ & $3.31 \mathrm{e} 1(10)$ \\
& 4 & 1,820 & 21 & 15.0 & 236 & 236 & $2.60 \mathrm{e} 1$ & $1.54 \mathrm{e} 2(12)$ \\
& 5 & 6,188 & 25 & 18.6 & 282 & 282 & $1.14 \mathrm{e} 2$ & $5.72 \mathrm{e} 2(13)$ \\
\hline \multirow{3}{*}{20} & 2 & 231 & 16 & 9.4 & 281 & 206 & $2.01 \mathrm{e} 1$ & $1.72 \mathrm{e} 1(8)$ \\
& 3 & 1,771 & 23 & 12.3 & 399 & 399 & $6.28 \mathrm{e} 1$ & $1.70 \mathrm{e} 2(10)$ \\
& 4 & 10,626 & 26 & 15.4 & 454 & 454 & $4.11 \mathrm{e} 2$ & $1.18 \mathrm{e} 3(12)$ \\
\hline
\end{tabular}

TABLE 8

Results for Example 5.2 with $\sigma=0.3$ and grid level 8: MultiRB solver versus standard $C G$.

\begin{tabular}{|c|rr|rr|rrr||c|}
\hline$m$ & $p$ & $n_{\xi}$ & $k$ & Inner & $n_{k}$ & Rank & Time & CG time (its) \\
\hline \multirow{4}{*}{8} & 2 & 45 & 17 & 9.8 & 128 & 45 & $3.21 \mathrm{e} 1$ & $1.34 \mathrm{e} 1(8)$ \\
& 3 & 165 & 21 & 12.2 & 160 & 129 & $4.14 \mathrm{e} 1$ & $5.66 \mathrm{e} 1(10)$ \\
& 4 & 495 & 24 & 14.5 & 183 & 178 & $5.11 \mathrm{e} 1$ & $1.97 \mathrm{e} 2(12)$ \\
& 5 & 1,287 & 27 & 16.9 & 207 & 207 & $6.40 \mathrm{e} 1$ & $5.53 \mathrm{e} 2(13)$ \\
\hline \multirow{3}{*}{12} & 2 & 91 & 15 & 9.9 & 165 & 89 & $4.78 \mathrm{e} 1$ & $3.00 \mathrm{e} 1(8)$ \\
& 3 & 455 & 18 & 12.2 & 201 & 196 & $6.16 \mathrm{e} 1$ & $1.75 \mathrm{e} 2(10)$ \\
& 4 & 1,820 & 21 & 15.0 & 236 & 236 & $8.64 \mathrm{e} 1$ & $8.21 \mathrm{e} 2(12)$ \\
& 5 & 6,188 & 25 & 18.6 & 281 & 281 & $1.88 \mathrm{e} 2$ & $3.07 \mathrm{e} 3(13)$ \\
\hline \multirow{3}{*}{20} & 2 & 231 & 16 & 9.4 & 281 & 206 & $1.11 \mathrm{e} 2$ & $9.47 \mathrm{e} 1(8)$ \\
& 3 & 1,771 & 23 & 12.3 & 399 & 399 & $1.97 \mathrm{e} 2$ & $8.45 \mathrm{e} 2(10)$ \\
& 4 & 10,626 & 26 & 15.4 & 454 & 454 & $5.56 \mathrm{e} 2$ & Out of Memory \\
\hline
\end{tabular}

number of random variables $m$ increase. As $\sigma$ and $m$ increase, the variance of the truncated diffusion coefficient increases, so it is intuitive that a richer approximation space would then be needed.

Adopting the parameter-free implementation for simplicity, we now compare the efficiency of using the MultiRB method to solve the matrix equation (3.5) and using CG to solve (3.6). Results obtained for grid levels 7 and 8 are presented in Tables 7 and 8, respectively. Here, we consider only the more challenging case $\sigma=0.3$. Comparing the results in Tables 7 and 8 we see that the dimension $n_{k}$ of the approximation space built by the MultiRB solver is independent of $h$, as predicted. The rank of the final approximation is also observed to be independent of $h$. Once again, when $n_{\xi}$ is small there is nothing to be gained by using the low-rank solver. Using CG on the Kronecker formulation is quicker and uses comparable memory. However, when $n_{\xi}$ is large (typically $n_{\xi}>100$ ) using the reduced basis solver on the matrix formulation is much quicker and uses substantially less memory. Indeed, in the largest experiment, where $n_{\xi}=10,626$ and the grid level is 8 , the Kronecker formulation consists of 690 million equations. We run out of memory when we apply CG to (3.6), but our new method is able to solve the matrix formulation of the system easily.

For completeness, we investigate the numerical ranks of the iterates generated when we apply CG to (3.6). In Table 9 , we report the rank of the $n_{x} \times n_{\xi}$ matrix $X_{j}$ associated with the $j$ th CG iterate as the iteration proceeds for the case $m=8, p=5$, and grid level 7. In this case, note that the largest possible rank is equal to $n_{\xi}=1,287$ (the number of columns of $X_{j}$ ). More precisely, at the $j$ th iteration we record the number of singular values $\sigma_{i}\left(X_{j}\right)$ satisfying $\sigma_{i}\left(X_{j}\right) / \sigma_{1}>\delta / n_{\xi}$ for $\delta=10^{-5}, 10^{-12}$. 
TABLE 9

Number of singular values $\sigma_{i}\left(X_{j}\right)$ of the $C G$ approximate solution matrix $X_{j}$ satisfying $\sigma_{i}\left(X_{j}\right) / \sigma_{1}>\delta / n_{\xi}$.

\begin{tabular}{|l|rrrrrrrrrrrrr|}
\hline it $j$ & 1 & 2 & 3 & 4 & 5 & 6 & 7 & 8 & 9 & 10 & 11 & 12 & 13 \\
\hline$\delta=10^{-5}$ & 1 & 9 & 45 & 96 & 140 & 172 & 185 & 194 & 193 & 202 & 204 & 204 & 204 \\
$\delta=10^{-12}$ & 1 & 9 & 45 & 165 & 359 & 462 & 529 & 603 & 645 & 711 & 750 & 791 & 810 \\
\hline
\end{tabular}

The first value of $\delta$ was used to determine the rank, equal to 207, reported in Table 7 for the final MultiRB iterate. The more stringent value $\delta=10^{-12}$ gives an idea of the numerical rank of the CG iterate. That is, the number of (relative) singular values above machine precision. Similar ranks are observed for the direction and residual matrices. The numbers reported in Table 9 illustrate that the CG iterates are not really of low rank. If a truncation procedure is implemented within CG to force the iterates to be of low rank, then orthogonality of the direction vectors is lost and the iteration error stagnates at a level that depends on the truncation threshold. The MultiRB algorithm builds up, a few columns at a time, an approximation $X_{k}$ of size $n_{x} \times n_{k}$ (in factored form) with rank $n_{k}=207$.

6. Summary and conclusions. This paper describes the design and implementation of the MultiRB method, an innovative solver for linear systems arising from stochastic Galerkin finite element approximation of elliptic PDEs with random coefficients. We consider the multiterm matrix equation formulation of the systems and develop a new solver that builds up an approximation space on-the-fly that exploits the inherent low-rank structure. The approximation space generated is inspired by ideas from rational approximation. Our numerical results demonstrate that highdimensional ( $>10^{9}$ unknowns) stochastic Galerkin linear systems can be solved in a few minutes on contemporary desktop computers. Moreover, the new solver requires significantly less memory than conventional mean-based preconditioned CG.

Appendix. Here we briefly explain how to perform the optional postprocessing step in Algorithm 4.1. Assuming that $Y_{k}=Y^{(1)}\left(Y^{(2)}\right)^{\top}$ with $Y^{(1)}, Y^{(2)}$ of rank $t$, the approximate solution to the original problem is also of rank $t$ and is given by $X_{k}=X^{(1)}\left(X^{(2)}\right)$ with $X^{(1)}:=V_{k} Y^{(1)}$ and $X^{(2)}:=\left(Y^{(2)}\right)^{\top}$. In practice, even if $Y_{k}$ is full rank it is interesting to approximate $Y_{k}$ by a low rank matrix $Y^{(1)}\left(Y^{(2)}\right)^{\top}$ that retains the largest singular values of $Y_{k}$ without affecting the overall approximation. For ease of exposition we assume that $n_{k} \leq n_{\xi}$. Let $Y_{k}=U \Theta Q^{\top}$ be the economy-size singular value decomposition of $Y_{k}$, that is, $Y_{k}=\sum_{r=1}^{n_{k}} \theta_{r} \mathbf{u}_{r} \mathbf{q}_{r}^{\top}$, where the values $\theta_{r}$ are sorted decreasingly. Suppose that $\theta_{\hat{r}+1}$ is the first singular value satisfying

$$
\theta_{\hat{r}+1} \leq \theta_{1} \frac{\text { tol_outer }}{n_{\xi}}
$$

and let $U_{\hat{r}}=\left[\mathbf{u}_{1}, \ldots, \mathbf{u}_{\hat{r}}\right], \Theta_{\hat{r}}=\operatorname{diag}\left(\theta_{1}, \ldots, \theta_{\hat{r}}\right)$ and $Q_{\hat{r}}=\left[\mathbf{q}_{1}, \ldots, \mathbf{q}_{\hat{r}}\right]$. Then $Y_{k}$ can be approximated by $Y^{(1)}\left(Y^{(2)}\right)^{\top}$ with $Y^{(1)}=U_{\hat{r}} \Theta_{\hat{r}}$ and $Y^{(2)}=Q_{\hat{r}}$, where the error is given by

$$
\left\|Y_{k}-Y^{(1)}\left(Y^{(2)}\right)^{\top}\right\|_{2}=\theta_{\hat{r}+1}
$$

(see Golub and Van Loan [11]). Note that this does not affect the final approximation in a significant manner. To show this, let us write $Y_{k}=Y^{(1)}\left(Y^{(2)}\right)^{\top}+E$ with $\|E\|_{2}=\theta_{\hat{r}+1}$ where $\theta_{\hat{r}+1}$ satisfies $(6.1)$ and recall that $\left\|Y_{k}\right\|_{2}=\left\|Y^{(1)}\left(Y^{(2)}\right)^{\top}\right\|_{2}=\theta_{1}$, $\left\|Y_{k}\right\|_{F}=\left(\theta_{1}^{2}+\cdots+\theta_{n_{k}}^{2}\right)^{1 / 2} \geq\left\|Y^{(1)}\left(Y^{(2)}\right)^{\top}\right\|_{F}$ and $\|E\|_{F} \leq \sqrt{n_{\xi}}\|E\|_{2}$. In this case 
we have that

$$
\begin{aligned}
& \frac{\left\|Y^{(1)}\left(Y^{(2)}\right)^{\top}-\left[Y_{k-1} ; 0\right]\right\|_{F}}{\left\|Y^{(1)}\left(Y^{(2)}\right)^{\top}\right\|_{F}} \\
& \quad=\frac{\left\|\left(Y_{k}-E\right)-\left[Y_{k-1} ; 0\right]\right\|_{F}}{\left\|Y^{(1)}\left(Y^{(2)}\right)^{\top}\right\|_{F}} \\
& \leq \frac{\left\|Y_{k}-\left[Y_{k-1} ; 0\right]\right\|_{F}}{\left\|Y^{(1)}\left(Y^{(2)}\right)^{\top}\right\|_{F}}+\frac{\|E\|_{F}}{\left\|Y^{(1)}\left(Y^{(2)}\right)^{\top}\right\|_{F}} \\
& \leq \frac{\left\|Y_{k}-\left[Y_{k-1} ; 0\right]\right\|_{F}}{\left\|Y_{k}\right\|_{F}}+\frac{\sqrt{n_{\xi}}\|E\|_{2}}{\left\|Y^{(1)}\left(Y^{(2)}\right)^{\top}\right\|_{F}} \\
& \leq \text { tol_outer }+\frac{\theta_{1} \text { tol_outer }}{\sqrt{n_{\xi}}\left\|Y^{(1)}\left(Y^{(2)}\right)^{\top}\right\|_{2}}=\left(1+\frac{1}{\sqrt{n_{\xi}}}\right) \cdot \text { tol_outer. }
\end{aligned}
$$

Hence, the new approximation satisfies the same stopping criterion with a value of the tolerance which approaches tol_outer as $n_{\xi}$ increases.

\section{REFERENCES}

[1] I. BabušKa, R. Tempone, And G.E. Zouraris, Galerkin finite element approximations of stochastic elliptic partial differential equations, SIAM J. Numer. Anal., 42 (2004), pp. 800825.

[2] J. Ballani And L. Grasedyck, A projection method to solve linear systems in tensor format, Numer. Linear Algebra Appl., 20 (2013), pp. 27-43.

[3] B. Beckermann And L. Reichel, Error estimation and evaluation of matrix functions via the Faber transform, SIAM J. Numer. Anal., 47 (2009), pp. 3849-3883.

[4] M.K. Deb, I.M. BABuŠKa, And J.T. Oden, Solution of stochastic partial differential equations using Galerkin finite element techniques, Comput. Methods Appl. Mech. Engrg., 190 (2001), pp. 6359-6372.

[5] V. Druskin, C. Lieberman, and M. Zaslavsky, On adaptive choice of shifts in rational Krylov subspace reduction of evolutionary problems, SIAM J. Sci. Comput., 32 (2010), pp. 2485-2496.

[6] V. Druskin and V. Simoncini, Adaptive rational Krylov subspaces for large-scale dynamical systems, Systems Control Lett., 60 (2011), pp. 546-560.

[7] M. Eigel, C.J. Gittelson, C. Schwab, and E. Zander, Adaptive stochastic Galerkin FEM, Comput. Methods Appl. Mech. Engrg, 270 (2014), pp. 247-269.

[8] H. Elman and D. Furnival, Solving the stochastic steady-state diffusion problem using multigrid, IMA J. Numer. Anal., 27 (2007), pp. 675-688.

[9] O.G. Ernst and E. Ullmann, Stochastic Galerkin matrices, SIAM J. Matrix Anal. Appl., 31 (2009/10), pp. 1848-1872.

[10] R.G. Ghanem and R.M. Kruger, Numerical solution of spectral stochastic finite element systems, Comput. Methods Appl. Mech. Engrg., 129 (1996), pp. 289-303.

[11] G.H. Golub and C.F. VAn Loan, Matrix Computations, John Hopkins University Press, Baltimore, MD, 1996.

[12] S. GütTEL, Rational Krylov approximation of matrix functions: Numerical methods and optimal pole selection, GAMM-Mitt., 36 (2013), pp. 8-31.

[13] R.A. Horn and C.R. Johnson, Matrix Analysis, Cambridge University Press, Cambridge, 1985.

[14] B. Khoromskij And C. Schwab, Tensor-structured Galerkin approximation of parametric and stochastic elliptic PDEs, SIAM J. Sci. Comput., 33 (2011), pp. 364-385.

[15] G.J. Lord, C.E. Powell, And T. Shardlow, An Introduction to Computational Stochastic PDEs, Cambridge University Press, Cambridge, 2014.

[16] A. Lu AND E.L. WACHSPREss, Solution of Lyapunov equations by Alternating Direction Implicit iteration, Comput. Math. Appl., 21 (1991), pp. 43-58.

[17] H.G. Matthies AND A. Keese, Galerkin methods for linear and nonlinear elliptic stochastic partial differential equations, Comput. Methods Appl. Mech. Engrg., 194 (2005), pp. 12951331 .

Copyright $@$ by SIAM. Unauthorized reproduction of this article is prohibited. 
[18] H.G. Matthies And E. ZAnder, Solving stochastic systems with low-rank tensor compression, Linear Algebra Appl., 436 (2012), pp. 3819-3838.

[19] C.E. Powell And H.C. Elman, Block-diagonal preconditioning for spectral stochastic finiteelement systems, IMA J. Numer. Anal., 29 (2009), pp. 350-375.

[20] A. Quarteroni, A. Manzoni, and F. Negri, Reduced Basis Methods for Partial Differential Equations, An Introduction, Springer, Switzerland, 2016.

[21] E. Rosseel and S. Vandewalle, Iterative solvers for the stochastic finite element method, SIAM J. Sci. Comput., 32 (2010), pp. 372-397.

[22] J. Sabino, Solution of Large-Scale Lyapunov Equations via the Block Modified Smith Method, Ph.D. thesis, Rice University, Houston, 2006.

[23] D.J. Silvester, A. Bespalov, And C.E. Powell, S-IFISS version 1.02, July 2015; available online at http://www.manchester.ac.uk/ifiss/s-ifiss1.0.tar.gz.

[24] V. Simoncini, Computational methods for linear matrix equations, SIAM Rev., 58 (2016), pp. $377-441$.

[25] B. Sousedik, R.G. Ghanem, And E.T. Phipps, Hierarchical Schur complement preconditioner for the stochastic Galerkin finite element methods, Numer. Linear Algebra Appl., 21 (2014), pp. $136-151$.

[26] E. Ullmann, A Kronecker product preconditioner for stochastic Galerkin finite element discretizations, SIAM J. Sci. Comput., 32 (2010), pp. 923-946.

Copyright (c) by SIAM. Unauthorized reproduction of this article is prohibited. 